\title{
ENDOMORPHISMS OF RELATIVELY FREE ALGEBRAS WITH WEAK EXCHANGE PROPERTIES
}

\author{
JOHN FOUNTAIN AND VICTORIA GOULD
}

\begin{abstract}
The structure of the endomorphism monoid of a stable basis algebra $A$ is described. It is shown to be an abundant monoid; the subsemigroup of endomorphisms of finite rank has a regular semigroup of left quotients.
\end{abstract}

This is a revised version of the article that appeared in Algebra Universalis 51 (2004), 257-285. The authors are grateful to Professor George Bergman for correcting their original definition of 'constant subalgebra'.

\section{INTRODUCTION}

The basis algebras of [11] generalise independence algebras (see [24], [14] and [3]). They stand in relation to independence algebras roughly as free modules over left Ore integral domains do to vector spaces. From the universal algebra perspective, basis algebras arise from the study of the interactions of various notions of independence and relative freeness, and this point of view is explored in some detail in our earlier paper [11]. In this paper we study endomorphisms of stable basis algebras, concentrating on describing the structure of the endomorphism monoid of such an algebra $A$. When $A$ is an independence algebra, the endomorphism monoid is regular with a particularly nice structure and one can use the structure, for example, to describe the idempotent generated submonoid. In [7], the structure of the multiplicative monoid of $n \times n$ integer matrices was used to find the submonoid generated by the idempotents, rediscovering a result of Laffey [19]. The approach of [7] was extended in [25] to the endomorphism monoid of free modules of finite rank over Hermite domains. Our aim is to find a common framework for these results, the first step being the analysis of the endomorphism monoid of a stable basis algebra. This is accomplished in the present paper; in a subsequent paper [12], we use the results obtained here to investigate the idempotent generated submonoid of such an endomorphism monoid.

In Section 2 we give a brief summary of the basic definitions and results connected with basis algebras. A fuller account can be found in [11]. We also prove some technical results needed for this paper. In particular we note that associated with each subalgebra of a basis algebra is a cardinal number called the rank of the subalgebra.

Central to the description of the endomorphisms of a stable basis algebra is the notion of abundant semigroup. This is the semigroup theory analogue of a PP ring, that is,

Date: August 24, 2004.

2000 Mathematics Subject Classification. Primary: 20M20, 08A35; Secondary 20M10, 08 A05.

Key words and phrases. basis, exchange property, endomorphism monoid, abundant, order, quotients. 
a ring with identity in which every principal one-sided ideal is projective. Indeed, the multiplicative semigroups of PP rings provide examples of abundant semigroups (see [22, Corollary I.3.4]), and one can characterise abundant monoids as those monoids in which each principal one-sided ideal is a projective act. For practical purposes, however, it is more useful to define abundant semigroups in terms of some generalisations of Green's relations $\mathscr{L}$ and $\mathscr{R}$, namely the relations $\mathscr{L}^{*}$ and $\mathscr{R}^{*}$. On a semigroup $S$, the relation $\mathscr{L}^{*}$ is defined by the rule that $a \mathscr{L}^{*} b$ if and only if the elements $a, b$ of $S$ are related by Green's relation $\mathscr{L}$ in some oversemigroup of $S$. The relation $\mathscr{R}^{*}$ is defined dually. A semigroup in which each $\mathscr{L}^{*}$-class and each $\mathscr{R}^{*}$-class contains an idempotent is said to be abundant. All the relevant definitions and results about abundant semigroups are collected together for easy reference in Section 3. In Section 4 we examine the $\mathscr{L}^{*}$-classes and the $\mathscr{R}^{*}$-classes of the endomorphism monoid End $A$ of a basis algebra $A$. In one of the main results of the paper (Theorem 4.9), we show that the endomorphism monoid of a basis algebra $A$ is abundant if and only if $A$ is stable. Moreover, when this is the case, End $A$ enjoys additional properties similar to those of regular semigroups.

We can say more about the subsemigroup $\operatorname{End}_{f} A$ of endomorphisms of finite rank, where the rank of an endomorphism is the rank of its image. Here we use the notion of a semigroup of left quotients [15], another concept inspired by a well known idea from ring theory, namely the classical ring of left quotients [2]. In many cases, but not all, these rings do provide examples of semigroups of left quotients [8]. In a ring $Q$ of left quotients of a ring $R$, the idea is that every non zero divisor in $R$ has an inverse in $Q$ and that every element of $Q$ is a left fraction of elements of $R$. In the semigroup case, for $Q$ to be a semigroup of left quotients of a semigroup $S$, we want every element of $Q$ to be a left fraction of elements of $S$, and we also want every element of $S$ which could be in a subgroup of an oversemigroup to be in a subgroup of $Q$. The semigroup $\operatorname{End}_{f} A$ is abundant and using results of [16], we can show that it has a regular semigroup of left quotients. Details about semigroups of left quotients [15], and the required results from [16] are given in Section 5, and the necessary analysis for applying these results to $\operatorname{End}_{f} A$ is in Section 6 .

A $*$-ideal of a semigroup is an ideal which is $\mathscr{L}^{*}$-saturated and $\mathscr{R}^{*}$-saturated. In the final section, we show that the $*$-ideals of End $A$, where $A$ is a stable basis algebra, are precisely the subsets $T_{\kappa}$ where $\kappa$ is a cardinal number and $T_{\kappa}$ is the set of all endomorphisms of rank

less than $\kappa$. For a positive integer $n$, the Rees quotient $T_{n} / T_{n-1}$ is a primitive abundant semigroup, and is isomorphic to a Rees matrix semigroup over a cancellative left Ore monoid. We give an explicit construction of an appropriate Rees matrix semigroup.

\section{Basis Algebras}

To make the paper as self contained as possible, we give a brief account of some basic ideas from [10] about independence, and introduce various classes of algebras culminating in stable basis algebras. For the fundamental concepts of universal algebra we refer the reader to [4], [17] or [23]. However, there are substantial differences in terminology and notation in these books. In the interest of clarity we begin by describing that adopted in this paper. By an algebra $A$ we mean an algebra in the sense of universal algebra. Thus $A$ 
comes equipped with a set (which may be empty) of basic operations all of which we assume to have finite arity. We permit 0 as an arity and refer to the images of nullary operations as constants. The operations on $A$ derived from the basic operations and projections by composition are called term operations. It is convenient to allow $\emptyset$ to be a subalgebra in the case where $A$ has no constants. Thus $\langle\emptyset\rangle$, the least subalgebra of $A$, is empty if and only if $A$ has no constants. On the other hand, if $A$ has constants, then $\langle\emptyset\rangle$ is the subalgebra of $A$ they generate. We say that a subalgebra $B$ of $A$ is nonconstant if $B \neq\langle\emptyset\rangle$, and refer to $\langle\emptyset\rangle$ as the constant subalgebra. It is worth noting that $c \alpha=c$ for every endomorphism $\alpha$ of $A$ and every element $c$ in the constant subalgebra. The definition of constant subablgebra has been modified since [11] was published. Further references in this article will be made to the revised version [10] of [11].

The key to many properties of the algebras we consider here is the interrelated behaviour of two closure operators. For the very special case of independence algebras, these closure operators coincide, but in general they are distinct.

Recall that a closure operator $C$ on a set $A$ is a function $C: \mathcal{P}(A) \rightarrow \mathcal{P}(A)$, where $\mathcal{P}(A)$ is the set of all subsets of $A$, such that for all $X, Y \in \mathcal{P}(A)$,

(1) $X \subseteq C(X)$;

(2) if $X \subseteq Y$, then $C(X) \subseteq C(Y)$;

(3) $C(C(X))=C(X)$.

A subset of $A$ of the form $C(X)$ is said to be closed. A closure operator $C$ is algebraic if for all $X \in \mathcal{P}(A)$,

$$
C(X)=\bigcup\{C(Y): Y \subseteq X, Y \text { is finite }\} .
$$

The canonical example of an algebraic closure operator is the subalgebra operator on an algebra $A$ which associates the subalgebra $\langle X\rangle$ to each subset $X$ of $A$.

If $A$ is a set with closure operator $C$, then a subset $X$ of $A$ is $C$-independent if for all $x \in X$,

$$
x \notin C(X \backslash\{x\}) .
$$

The exchange property (EP) for a closure operator $C$ on a set $A$ is defined as follows:

(EP) for all $x, y \in A$ and $X \subseteq A$,

$$
\text { if } x \in C(X \cup\{y\}) \text { and } x \notin C(X) \text {, then } y \in C(X \cup\{x\}) \text {. }
$$

Algebraic closure operators which satisfy the exchange property are intimately connected with abstract dependence relations, and we now restate several fundamental results from $[4$, Section VII.2] in terms of algebraic closure operators. The first comes from the proof of Proposition VII.2.1 in [4] (see also [23, p.50 Exercise 6(a)]).

Lemma 2.1. Let $C$ be an algebraic closure operator on a set $A$. Then the following conditions are equivalent:

(1) $C$ satisfies the exchange property,

(2) if $X$ is a $C$-independent subset of $A$ and $y \notin C(X)$, then $X \cup\{y\}$ is $C$-independent. 
Lemma 2.2. [4, Lemma VII.2.2] Let $C$ be an algebraic closure operator satisfying (EP) on a set $A$ and let $Y \subseteq X \subseteq A$. Then the following conditions are equivalent:

(1) $Y$ is a maximal $C$-independent subset of $X$,

(2) $Y$ is $C$-independent and $C(Y)=C(X)$,

(3) $Y$ is minimal with respect to $C(Y)=C(X)$.

If $C$ is an algebraic closure operator on a set $A$, then it is easy to see that the union of a chain of $C$-independent sets is $C$-independent. Since $\emptyset$ is clearly $C$-independent, a Zorn's lemma argument gives that, for any subset $X$ of $A$, there is a maximal $C$-independent subset of $X$.

Writing a slightly generalised version of Theorem VII.2.4 of [4] in terms of closure operators (see also [23, p.50 Exercise 6(b)]) we have the following result.

Theorem 2.3. Let $C$ be an algebraic closure operator satisfying (EP) on a set $A$, and let $X \subseteq Y \subseteq A$. If $X$ is $C$-independent, then there is a $C$-independent subset $Z$ with $X \subseteq Z \subseteq Y$ and $C(Z)=C(Y)$. Moreover, if $Z$ and $Z$ ' are $C$-independent subsets of $Y$ with $C(Z)=C\left(Z^{\prime}\right)=C(Y)$, then they have the same cardinality.

In view of Lemma 2.2, a $C$-independent subset $Z$ with $Z \subseteq Y$ and $C(Z)=C(Y)$ is a maximal $C$-independent subset of $Y$. Such a maximal $C$-independent subset is often called a $C$-basis of $Y$, but we reserve the term "basis" for a free basis. In view of Lemma 2.2 and Theorem 2.3, we can define the $C$-rank of $Y$ to be the cardinality of any maximal $C$-independent subset of $Y$. The following corollary is a straightforward consequence of Lemma 2.2 and Theorem 2.3.

Corollary 2.4. Let $C$ be an algebraic closure operator satisfying (EP) on a set $A$. If $X$ and $Y$ are subsets of $A$ with $X \subseteq Y$, then

(1) $C-\operatorname{rank}(X) \leqslant C-\operatorname{rank}(Y)$,

(2) $C$-rank $(X)=C-\operatorname{rank}(C(X))$.

We explain the what is meant by independence algebra $A$ later in the section when we have discussed $A$-freeness, but we mention now that one of the defining properties of such algebras is that the subalgebra operator satisfies (EP). This paper is concerned with wider classes of algebras satisfying corresponding conditions for a second closure operator, which we now describe.

For an element $a$ of an algebra $A$ and a subset $X$ of $A$ we say that $a$ depends on $X$ and write $a \prec X$ if

$$
a \in\langle\emptyset\rangle \text { or }\langle a\rangle \cap\langle X\rangle \neq\langle\emptyset\rangle \text {. }
$$

For subsets $X, Y$ of $A$ we say that $Y$ depends on $X$, and write $Y \prec X$, if $y \prec X$ for every $y \in Y$. Notice that for all $a \in A$ and subsets $X$ of $A$,

$$
\begin{gathered}
a \prec \emptyset \text { if and only if } a \in\langle\emptyset\rangle ; \\
a \prec X \text { if and only if } a \prec\langle X\rangle
\end{gathered}
$$

and

$$
c \prec X \text { for any } c \in\langle\emptyset\rangle \text {. }
$$


From the relation $\prec$ we obtain a second closure operator on an algebra $A$ which is crucial to our work.

For any subset $X$ of an algebra $A$ we let

$$
\mathrm{PC}(X)=\{a \in A: a \prec X\} .
$$

It is easy to see that $\langle X\rangle \subseteq \mathrm{PC}(X)$, and $\mathrm{PC}(X)=\mathrm{PC}(\langle X\rangle)$. From [10, Theorem 1.6] we have the following.

Lemma 2.5. Let $A$ be an algebra such that for all $a \in A$ and $X, Y \subseteq A$,

$$
\text { if } a \prec X \text { and } X \prec Y \text { then } a \prec Y \text {. }
$$

Then $\mathrm{PC}: \mathcal{P}(A) \rightarrow \mathcal{P}(A)$ is an algebraic closure operator.

Further, $\mathrm{PC}(X)$ is a subalgebra of $A$ for any subset $X$ of $A$.

An algebra $A$ is a weak exchange algebra if $A$ satisfies (T) and the closure operator PC satisfies (EP). The latter condition is called the weak exchange property (WEP) and says that for all $x, y \in A$ and $X \in \mathcal{P}(A)$, if

$$
\langle x\rangle \cap\langle X \cup\{y\}\rangle \neq\langle\emptyset\rangle \text { and }\langle x\rangle \cap\langle X\rangle=\langle\emptyset\rangle,
$$

then

$$
\langle y\rangle \cap\langle X \cup\{x\}\rangle \neq\langle\emptyset\rangle \text {. }
$$

If an algebra $A$ satisfies (EP) for the subalgebra operator, then by Lemma 1.8 of [10], (T) holds and moreover, $\mathrm{PC}(X)=\langle X\rangle$ for all $X \in \mathcal{P}(A)$, so that independence algebras are weak exchange algebras. Examples of weak exchange algebras which are not independence algebras are given in [10].

On a weak exchange algebra $A$ we have two notions of independence, one arising from the subalgebra operator and the other from the operator PC. A subset $X$ of $A$ is independent if it is independent with respect to the subalgebra operator, and directly independent if it is independent with respect to PC. As remarked in [10], every directly independent subset is independent.

If $A$ is a weak exchange algebra, then by Theorem 2.3, we can define the rank of a subset $X$ of $A$ with respect to $\mathrm{PC}$; it is the cardinality of any maximal directly independent subset of $X$. If $A$ also satisfies (EP) with respect to the subalgebra operator, we can also define the rank of $X$ (with respect to this operator) as the cardinality of any maximal independent subset of $X$. However, it follows from Lemma 1.8 of [10], that these two ranks are equal, and so there is no ambiguity when we refer to the rank of $X$ with respect to $\mathrm{PC}$ as simply the rank of $X$.

Notice that a subalgebra of a weak exchange algebra is a weak exchange algebra, and the notion of direct independence is independent of the subalgebra under consideration. From [10] we have the following.

Proposition 2.6. Let $X$ be a subset of a weak exchange algebra $A$. Then

(1) $\operatorname{rank}(\langle X\rangle)=\operatorname{rank}(X) \leqslant|X|$,

(2) if $X$ is finite and $\operatorname{rank}(\langle X\rangle)=|X|$, then $X$ is directly independent,

(3) if $B$ is a subalgebra of $A$, then $\operatorname{rank} B \leqslant \operatorname{rank} A$. 
We say that a subset $X$ of an algebra $A$ is pure, or (if there is any danger of ambiguity) pure in $A$, if for each element $a$ of $A$,

$$
a \prec X \text { implies that } a \in X \text {, }
$$

or equivalently,

$$
X=\mathrm{PC}(X) .
$$

Note that $\langle\emptyset\rangle$ is always pure, and for an algebra $A$ satisfying $(\mathrm{T})$, the pure subsets are precisely the closed sets of the closure operator PC.

From Lemma 2.2, we deduce

Corollary 2.7. A directly independent subset $X$ of a weak exchange algebra $A$ is maximal directly independent (equivalently, a PC-basis), if and only if $y \prec X$ for all $y \in A$.

The following elementary lemma will be useful.

Lemma 2.8. Let $X$ be a directly independent subset of a weak exchange algebra $A$, and for each $x \in X$, let $t_{x}$ be a unary term operation such that $t_{x}(x) \notin\langle\emptyset\rangle$. Then the elements $t_{x}(x)(x \in X)$ are distinct, and the set $\left\{t_{x}(x): x \in X\right\}$ is directly independent.

Proof. If $x, y \in X$ and $t_{x}(x)=t_{y}(y)$, then $\langle x\rangle \cap\langle y\rangle \neq\langle\emptyset\rangle$ so that $x=y$, since $X$ is directly independent.

If $\left\langle t_{x}(x)\right\rangle \cap\left\langle\left\{t_{y}(y): y \in X \backslash\{x\}\right\}\right\rangle \neq\langle\emptyset\rangle$, then, clearly, $\langle x\rangle \cap\langle X \backslash\{x\}\rangle \neq\langle\emptyset\rangle$, a contradiction.

Corollary 2.9. Let $A$ be a weak exchange algebra. If $B$ and $D$ are subalgebras of $A$ with $\mathrm{PC}(B)=\mathrm{PC}(D)$, then $\mathrm{PC}(B \cap D)=\mathrm{PC}(B)$.

Proof. Let $X$ and $Y$ be PC-bases of $B$ and $D$ respectively. From Lemma 2.2, $\mathrm{PC}(B)=$ $\mathrm{PC}(X)$ and $\mathrm{PC}(D)=\mathrm{PC}(Y)$ so that in particular, $Y \prec X$. Hence, if $y \in Y$, then $\langle y\rangle \cap\langle X\rangle \neq\langle\emptyset\rangle$. Therefore, there is a term operation $t_{y}$ such that $t_{y}(y) \in\langle X\rangle$ and $t_{y}(y)$ is not in $\langle\emptyset\rangle$. By Lemma 2.8, the set $U=\left\{t_{y}(y): y \in Y\right\}$ is directly independent, and $t_{y}(y) \neq t_{z}(z)$ for $y, z \in Y$ with $y \neq z$. Note that $U \subseteq B \cap D$ and that $Y \prec U$ so that $\mathrm{PC}(D) \prec U$ by condition (T). Thus $\mathrm{PC}(D) \subseteq \mathrm{PC}(U)$ and since $\mathrm{PC}$ is a closure operator,

$$
\mathrm{PC}(U) \subseteq \mathrm{PC}(B \cap D) \subseteq \mathrm{PC}(D) \subseteq \mathrm{PC}(U) .
$$

Hence $\mathrm{PC}(B \cap D)=\mathrm{PC}(B)$.

A third property related to notions of independence is that of being $A$-free. A subset $X$ of $A$ is $A$-free if every function from $X$ to $A$ can be extended to a morphism from $\langle X\rangle$ to $A$. If $A=\langle X\rangle$ for some $A$-free set $X$, then $A$ is relatively free.

An algebra $A$ is an independence algebra if the subalgebra operator satisfies (EP) and any independent subset of $A$ is $A$-free. This paper is concerned with weak exchange algebras satisfying corresponding conditions for the closure operator PC.

Following [10] we say that an algebra $A$ is a weak independence algebra if it is a weak exchange algebra such that every directly independent subset is $A$-free. We will see that for a special class of weak independence algebras that we now describe, the $A$-free subsets in $A$ are precisely the directly independent subsets. 
A useful fact about unary term operations on a weak independence algebra is given in the following result from [10, Proposition 5.2].

Proposition 2.10. If $A$ is a nonconstant weak independence algebra such that $\emptyset \neq\langle\emptyset\rangle$, then, for a unary term operation $t$, the following are equivalent:

(1) $t=\kappa_{c}$ for some $c \in A$,

(2) $t(a) \in\langle\emptyset\rangle$ for all $a \in A$,

(3) $t(x) \in\langle\emptyset\rangle$ for some $x \in A \backslash\langle\emptyset\rangle$.

Let $A$ be an algebra and let $T_{1}$ be the set of all unary term operations on $A$. Clearly, $T_{1}$ is a monoid under composition of functions. We let

$$
T_{C}=\left\{\kappa_{c}: c \in\langle\emptyset\rangle\right\}
$$

so that if $A$ has no constants, then $T_{C}=\emptyset$. In [10], Proposition 2.10 is used to show that if $A$ is a nonconstant weak independence algebra, then $T_{1}^{*}=T_{1} \backslash T_{C}$ is a right cancellative, left Ore submonoid of the monoid of all unary term operations on $A$.

We say that a nonconstant weak independence algebra $A$ is torsion-free if for any $t \in T_{1}^{*}$ and elements $a, b \in A$,

$$
\text { if } t(a)=t(b) \text {, then } a=b .
$$

We declare a constant algebra to be torsion-free.

It is noted in [10] that if $A$ is a nonconstant torsion-free weak independence algebra, then the monoid $T_{1}^{*}$ is cancellative.

By [10, Lemma 5.6] we have that, in a nonconstant torsion-free weak independence algebra $A$, the $A$-free subsets are precisely the directly independent subsets. Certainly, subalgebras of weak independence algebras have the same property. We need the following results from [10].

Lemma 2.11. [10, Corollary 5.8] Let $B$ be a nonconstant subalgebra of a torsion-free weak independence algebra $A$. Then $B$-free subsets of $B$ are $A$-free.

Lemma 2.12. [10, Lemma 4.3] Let $X$ be a directly independent subset of a weak independence algebra $A$, and let $\alpha: X \rightarrow A$ be one-one. If $X \alpha$ is directly independent, then the morphism $\bar{\alpha}:\langle X\rangle \rightarrow A$ which extends $\alpha$ is one-one.

Corollary 2.13. [10, Corollary 4.4] Let $A$ be a weak independence algebra. If $X$ and $Y$ are directly independent subsets of $A$ of the same cardinality, then the subalgebras $\langle X\rangle$ and $\langle Y\rangle$ are isomorphic.

Proposition 2.14. [10, Proposition 4.2] Let $B$ be a subalgebra of a weak independence algebra $A$ and $\theta: B \rightarrow A$ be a morphism. Then

(1) if $\theta$ is one-one and $X \subseteq B$ is directly independent, then $X \theta$ is directly independent;

(2) if $Y$ is a directly independent subset of $B \theta$ and $Z \subseteq B$ is such that $Z \theta=Y$ and $\theta$ is one-one on $Z$, then $Z$ is directly independent.

Corollary 2.15. Isomorphic subalgebras of a weak independence algebra have the same rank. 
The following remark, stated as a lemma, will also be useful.

Lemma 2.16. Let $B$ be a subalgebra of a weak independence algebra $A$ and $\theta: B \rightarrow A$ be a one-one morphism. Let $b \in B$ and $Y \subseteq B$. If $b \prec Y$, then $b \theta \prec Y \theta$.

Let $A$ be an algebra. A basis of $A$ is an $A$-free set $X \subseteq A \backslash\langle\emptyset\rangle$ such that $X$ generates $A$. From the definition of torsion-free weak independence algebra and [10, Lemma 5.6], a basis in such an algebra $A$ is the same thing as a generating set which is directly independent. Moreover, from Corollary 2.7, a basis is certainly a PC-basis. We remark that $\langle\emptyset\rangle$ has unique basis $\emptyset$. In general, not all subalgebras of $A$ will have a basis, but by Proposition 2.6, if $Y$ is a set of generators for a subalgebra $B$, then the rank of $B$ is at most $|Y|$. If $Y$ is actually a basis of $B$, then the rank of $B$ is $|Y|$.

From [10] we have

Proposition 2.17. Let $A$ be a torsion-free weak independence algebra with basis $X$. If $Y$ is a subset of $X$, then $\langle Y\rangle$ is pure.

We now define a basis algebra to be a torsion-free weak independence algebra $A$ which satisfies the following condition:

(PEP): if $P, Q$ are pure subalgebras in $A$ with $P \subseteq Q$, and $X$ is a basis for $P$, then there is a basis $Y$ for $Q$ with $X \subseteq Y$.

Condition (PEP) may be regarded as a converse to Proposition 2.17.

Since $\langle\emptyset\rangle$ is a pure subalgebra of $A$ with basis $\emptyset$, it follows that if $P$ is a pure subalgebra of $A$, then it has a basis, that is, it is relatively free. In fact, by [10, Proposition 8.2], $P$ is itself a basis algebra. We emphasise that, as $A$ is certainly pure, it is itself relatively free.

Lemma 2.18. [10, Proposition 8.2] Let $B$ be a subalgebra of a basis algebra $A$. If $B$ has a basis, then $B$ is isomorphic to $\mathrm{PC}(B)$, and $B$ is a basis algebra.

Let $\kappa$ be a cardinal. A basis algebra $A$ is $\kappa$-free if every subalgebra of $A$ having a generating set of cardinality at most $\kappa$ is relatively free, that is, has a basis. We say that $A$ is stable if it is $\kappa$-free for $\kappa=\operatorname{rank} A$.

A basis algebra is semihereditary if every finitely generated subalgebra is a basis algebra and hereditary if every subalgebra is a basis algebra. In the examples below, we see that not every semihereditary basis algebra is hereditary.

From Lemma 2.18, a basis algebra is (semi)hereditary if and only if every (finitely generated) subalgebra has a basis.

We conclude this section by recalling the three main known examples of basis algebras.

First, we have free modules of finite rank over a Bezout domain where a Bezout domain is an integral domain in which every finitely generated one-sided ideal is principal. Such rings can also be characterised as domains $R$ such that for every matrix $A$ over $R$, there are invertible matrices $P, Q$ over $R$ with $P A$ and $A Q$ being upper triangular. An example of a commutative Bezout domain which has some nonfinitely generated ideals is the subring of $\mathbb{Q}[x]$ consisting all polynomials with integer constant term. From [10], finitely generated free modules over a Bezout domain are semihereditary basis algebras, and if the domain $R$ is a principal ideal domain, then such modules are hereditary basis algebras. 
The endomorphism monoid of a free module of rank $n$ over any ring $R$ is, of course, just the multiplicative monoid of all $n \times n$ matrices over $R$. In [7] the first author investigated the structure of such monoids; the results therein motivate this paper.

Recall that for a monoid $T$, a (left) $T$-act is a set $A$ on which $T$ acts unitarily, that is, for all $t \in T$ and all $a \in A$, there is a uniquely defined element $t a$ of $A$ and

(1) $1 a=a$ for all $a \in A$,

(2) $(s t) a=s(t a)$ for all $a \in A$ and $s, t \in T$.

The free $T$-act $F_{X}$ with free generating set $X$ is the disjoint union $\coprod_{x \in X} T x$ where each $T x$ is isomorphic to the left $T$-act $T$ via an isomorphism which takes $x$ to 1 . (This can also be viewed as the set $T \times X$ with $T$-action $t(s, x)=(t s, x)$ for $s, t \in T$ and $x \in X$.) The monoid of endomorphisms of $F_{X}$ can be described as a wreath product (see [21, Construction II.7.6 and Theorem II.7.7]). The monoid of all self-maps (acting on the right of their arguments) of a non-empty set $X$ is denoted by $T(X)$. For a monoid $T$ and non-empty set $X$, we denote by $W\left(T^{X}\right)$ the monoid with universe $T^{X} \times T(X)$ and multiplication given by

$$
(f, \alpha)(g, \beta)=\left(f^{\alpha} g, \alpha \beta\right)
$$

where $x\left(f^{\alpha} g\right)=(x f)((x \alpha) g)$ for each $x \in X$. The identity element of $W\left(T^{X}\right)$ is $\left(c_{1}, I_{X}\right)$ where $c_{1}$ is the constant function from $X$ to $T$ with value 1 . It is straightforward to verify that the mapping which associates with the endomorphism $\theta$ the element $\left(f_{\theta}, \alpha_{\theta}\right)$ determined by $(1, x) \theta=\left(x f_{\theta}, x \alpha_{\theta}\right)$ is a monoid isomorphism.

A free $T$-act on $X$ is a semihereditary basis algebra when $X$ is finite and $T$ is a cancellative monoid in which every finitely generated left ideal is principal; it is hereditary if every left ideal is principal.

Independence algebras provide our third class of basis algebras. Recall that an independence algebra is an algebra $A$ for which the subalgebra operator satisfies the exchange property and in which every independent generating set is $A$-free. It is pointed out in [10] that every independence algebra is a hereditary basis algebra. The structure of the endomorphism monoid of an independence algebra is described in [14].

\section{Abundant Semigroups}

We recall the concepts and results of semigroup theory that are related to abundant semigroups and which we need to describe the structure of an endomorphism monoid of a basis algebra. As far as possible, we follow the notation and terminology of [18].

We have already introduced the notation $T(X)$ for the monoid of all self-maps of $X$ written on the right of their arguments. When the mappings are written on the left of their arguments we use the notation $T^{*}(X)$. For a semigroup $S$ we define $S^{1}$ to be the smallest monoid containing $S$ so that if $S$ is a monoid, $S=S^{1}$ and otherwise, $S^{1}=S \cup\{1\}$. The right regular representation of $S$ is the embedding $\rho: S \rightarrow T\left(S^{1}\right)$ given by a $\rho=\rho_{a}$ where $x \rho_{a}=x a$. Similarly, one has the left regular representation $\lambda: S \rightarrow T^{*}\left(S^{1}\right)$ where $a \lambda=\lambda_{a}$ is left multiplication by $a$.

We define reflexive, transitive relations $\leqslant \mathscr{R}^{*}$ and $\leqslant \mathscr{L}^{*}$ on a semigroup $S$ as follows. Let $a, b \in S$. Then $a \leqslant \mathscr{R}^{*} b$ if $\operatorname{Ker} \rho_{b} \subseteq \operatorname{Ker} \rho_{a}$, and $a \leqslant \mathscr{L}^{*} b$ if $\operatorname{Ker} \lambda_{b} \subseteq \operatorname{Ker} \lambda_{a}$. Thus $a \leqslant \mathscr{R}^{*} b$ 
means that for all $x, y \in S^{1}$,

$$
x b=y b \quad \text { implies that } \quad x a=y a,
$$

and similarly for $\leqslant \mathscr{L}^{*}$.

The equivalence relations induced by $\leqslant \mathscr{R}^{*}$ and $\leqslant \mathscr{L}^{*}$ are denoted by $\mathscr{R}^{*}$ and $\mathscr{L}^{*}$ respectively, so that $a \mathscr{R}^{*} b$ if and only if $\operatorname{Ker} \rho_{a}=\operatorname{Ker} \rho_{b}$, and $a \mathscr{L}^{*} b$ if and only if $\operatorname{Ker} \lambda_{a}=\operatorname{Ker} \lambda_{b}$. Thus $a \mathscr{R}^{*} b$ means that for all $x, y \in S^{1}$,

$$
x a=y a \quad \text { if and only if } \quad x b=y b .
$$

We remark that the relation $\leqslant \mathscr{R}^{*}$ is left compatible with multiplication so that $\mathscr{R}^{*}$ is a left congruence on $S$; similarly, $\leqslant \mathscr{L}^{*}$ is right compatible, and $\mathscr{L}^{*}$ is a right congruence on $S$.

We define the relations $\mathscr{H}^{*}$ and $\mathscr{D}^{*}$ by putting $\mathscr{H}^{*}=\mathscr{R}^{*} \cap \mathscr{L}^{*}$ and $\mathscr{D}^{*}=\mathscr{R}^{*} \vee \mathscr{L}^{*}$.

The following easy result is useful and will be used without further reference.

Lemma 3.1. If $e$ is an idempotent of a semigroup $S$ and if $b \in S$ is such that $b \leqslant \mathscr{R}^{*} e$, then $e b=b$. In particular, e acts as a left identity within its $\mathscr{R}^{*}$-class.

Proof. Since $e e=1 e$, we have $e b=1 b=b$.

There is, of course, a dual result for $\leqslant \mathscr{L}^{*}$.

A semigroup $S$ is abundant if each $\mathscr{R}^{*}$-class and each $\mathscr{L}^{*}$-class contains an idempotent. For more details about the relations $\mathscr{L}^{*}, \mathscr{R}^{*}, \mathscr{H}^{*}$ and $\mathscr{D}^{*}$ and abundant semigroups we refer the reader to [6].

Green's relations $\mathscr{R}$ and $\mathscr{L}$ on a semigroup $S$ are the equivalence relations associated with the reflexive, transitive relations $\leqslant \mathscr{R}$ and $\leqslant \mathscr{L}$ respectively, where for elements $a, b$ of $S$,

and dually,

$$
a \leqslant \mathscr{R} b \text { if and only if } a S^{1} \subseteq b S^{1},
$$

$$
a \leqslant \mathscr{L} b \quad \text { if and only if } \quad S^{1} a \subseteq S^{1} b .
$$

Thus

$$
a \mathscr{R} b \quad \text { if and only if } a S^{1}=b S^{1}
$$

and

$$
a \mathscr{L} b \quad \text { if and only if } \quad S^{1} a=S^{1} b .
$$

Notice that $a \mathscr{R} b$ if and only if there are elements $x, y$ of $S^{1}$ such that $a x=b$ and $b y=a$, and similarly for $\mathscr{L}$. The relation $\mathscr{D}$ is the join of $\mathscr{L}$ and $\mathscr{R}$, and since $\mathscr{L}$ and $\mathscr{R}$ commute (see [18, Proposition 2.1.3]), we have $\mathscr{D}=\mathscr{R} \circ \mathscr{L}=\mathscr{L} \circ \mathscr{R}$.

It is not difficult to show that, for any semigroup, we have $\leqslant \mathscr{R} \subseteq \leqslant \mathscr{R}^{*}$ and $\leqslant \mathscr{L} \subseteq \leqslant \mathscr{L}^{*}$ so that $\mathscr{R} \subseteq \mathscr{R}^{*}$ and $\mathscr{L} \subseteq \mathscr{L}^{*}$. In fact, we have the following characterisation of $\leqslant \mathscr{R}^{*}$ from $[20]$.

Lemma 3.2. Let $a, b$ be elements of a semigroup $S$. Then $a \leqslant \mathscr{R}^{*} b$ if and only if there is an oversemigroup $T$ of $S$ in which $a \leqslant \mathscr{R} b$; and $a \mathscr{R}^{*} b$ if and only if there is an oversemigroup $T$ of $S$ in which $a \mathscr{R} b$. 
There are, of course, corresponding dual results characterising $\leqslant \mathscr{L}^{*}$ and $\mathscr{L}^{*}$.

An element $a$ of a semigroup $S$ is regular if there is an element $b$ in $S$ such that $a b a=a$, and $S$ is regular if all its elements are regular. For regular elements of $S$, we have $a \mathscr{R} b$ if and only if $a \mathscr{R}^{*} b$, and $a \mathscr{L} b$ if and only if $a \mathscr{L}^{*} b$. Thus, on a regular semigroup, $\mathscr{L}=\mathscr{L}^{*}$ and $\mathscr{R}=\mathscr{R}^{*}$. It follows that every regular semigroup is abundant because it is well known that, in a regular semigroup, each $\mathscr{R}$-class and each $\mathscr{L}$-class contains an idempotent (see [18, Proposition 2.3.2]).

The standard notation for the $\mathscr{R}$-class (or $\mathscr{R}^{*}$-class) containing an element $a$ is $R_{a}$ (or $R_{a}^{*}$ ), and there is corresponding notation for the classes of the other equivalence relations we have introduced.

In a non-regular abundant semigroup $S$, the relationship between the idempotents in $R_{a}^{*}$ and those in $L_{a}^{*}$ for a given $a \in S$ need not be as strong as it is in a regular semigroup. In view of this, El Qallali and Fountain [5] introduced the notion of an idempotent connected (IC) abundant semigroup. Using [1, Lemma 2.3], we can define this concept as follows: An abundant semigroup $S$ is idempotent connected if for each element $a$ of $S$ and each idempotent $e$ (resp. $f$ ) with $e \leqslant \mathscr{L}^{*} a$ (resp. $f \leqslant \mathscr{R}^{*} a$ ), there is an element $b \in S$ (resp. $c \in S$ ) such that $a e=b a$ (resp. $f a=a c$ ). More details about IC abundant semigroups can be found in [1], [5] and [22]. For our purposes here, all that is needed is the following lemma which combines [7, Lemma 2.4] and [22, Proposition II.2.6], and which underlies our description of the structure of the endomorphism monoid of a basis algebra.

Lemma 3.3. If $S$ is a semigroup in which every $\mathscr{H}^{*}$-class contains a regular element, then $\mathscr{D}^{*}=\mathscr{R}^{*} \circ \mathscr{L}^{*}=\mathscr{L}^{*} \circ \mathscr{R}^{*}$, and $S$ is IC abundant.

An ideal $I$ of a semigroup $S$ is a $*$-ideal if it is a union of $\mathscr{R}^{*}$-classes and of $\mathscr{L}^{*}$-classes.

Lemma 3.4. Let $T$ be $a *$-ideal of an abundant semigroup $S$. If $a \in S$ and $b \in T$ with $a \leqslant \mathscr{R}^{*} b$ in $S$, then $a \in T$.

Proof. We know $b \mathscr{R}^{*} e \in E(S)$; thus $e \in E(T)$ since $T$ is closed under $\mathscr{R}^{*}$. Now $e a=a$ by Lemma 3.1 so that $a \in T$ since $T$ is an ideal.

The following technical lemma will be needed in Section 6 .

Lemma 3.5. Let $T$ be $a *$-ideal of an abundant semigroup $S$. Then for any $a, b \in T$,

$$
a \leqslant \mathscr{R}^{*} b \text { in } T \text { if and only if } a \leqslant \mathscr{R}^{*} b \text { in } S
$$

and dually,

$$
a \leqslant \mathscr{L}^{*} b \text { in } T \text { if and only if } a \leqslant \mathscr{L}^{*} b \text { in } S .
$$

Consequently, $T$ is abundant. Moreover, if every $\mathscr{H}^{*}$-class of $S$ contains a regular element, then so does every $\mathscr{H}^{*}$-class of $T$.

Let $U$ be a *-ideal of $S$ with $U \subseteq T$. Then for any non-zero elements $a, b$ in the Rees quotient $T / U$,

and

$$
a \leqslant \mathscr{R}^{*} b \text { in } T / U \text { if and only if } a \leqslant \mathscr{R}^{*} b \text { in } T
$$

$$
a \leqslant \mathscr{L}^{*} b \text { in } T / U \text { if and only if } a \leqslant \mathscr{R}^{*} b \text { in } T \text {. }
$$


Consequently, $T / U$ is abundant and if every $\mathscr{H}^{*}$-class of $T$ contains a regular element, then so does every $\mathscr{H}^{*}$-class of $T / U$.

Proof. From Lemma 3.2, if $a, b \in T$ and $a \leqslant \mathscr{R}^{*} b$ in $S$, certainly $a \leqslant \mathscr{R}^{*} b$ in $T$.

Suppose now that $a, b \in T$ and $a \leqslant \mathscr{R}^{*} b$ in $T$. Let $x, y \in S^{1}$ and suppose that $x b=y b$. Since $S$ is abundant and $T$ is a $*$-ideal, we have $b \mathscr{R}^{*} e$ in $S$ for some $e \in E(T)$. Thus $e b=b$ so that $e a=a$ also. Now

$$
(x e) b=x(e b)=x b=y b=y(e b)=(y e) b
$$

and $x e, y e \in T$ so that

$$
x a=x(e a)=(x e) a=(y e) a=y(e a)=y a
$$

and $a \leqslant \mathscr{R}^{*} b$ in $S$. The dual result holds for $\leqslant \mathscr{L}^{*}$.

If every $\mathscr{H}^{*}$-class of $S$ contains a regular element, then if $a \in T, a \mathscr{H}^{*} b$ for some $b \in S$ with $b=b c b$ for some $c \in S$. Now $T$ is a $*$-ideal, so that $b \in T$ and $a \mathscr{H}^{*} b$ in $T$. Also, $b=b(c b c) b$ and $c b c \in T$, so that $b$ is regular in $T$.

Let $U$ be as given. Let $a, b$ be non-zero elements of the Rees quotient $T / U$ and suppose that $a \leqslant \mathscr{R}^{*} b$ in $S$. Let $x, y \in(T / U)^{1}$ and suppose that $x b=y b$. If $x b=y b=0$ in $T / U$, then either $x=0$, in which case $x a=0$, or $x \neq 0$ and $x b \in U$. Now since $\leqslant \mathscr{R}^{*}$ is left compatible, $x a \leqslant \mathscr{R}^{*} x b$ in $S$ gives $x a \in U$ by Lemma 3.4. Thus in either case, $x a=0$ in $T / U$ and similarly, $y a=0$ in $T / U$.

Suppose now that $x b=y b \neq 0$ in $T / U$. We must then have $x, y \in(T \backslash U) \cup\{1\}$ and $x b=y b$ in $S$. But then $x a=y a$ in $S$, so that certainly $x a=y a$ in $T / U$.

Conversely, suppose that $a, b$ are non-zero elements of $T / U$ and $a \leqslant \mathscr{R}^{*} b$ in $T / U$. Let $e, f \in E(S)$ with $e \mathscr{R}^{*} a$ and $f \mathscr{R}^{*} b$ in $S$. Since $T, U$ are $*$-ideals, $e, f$ are non-zero elements of $T / U$ and from the above,

$$
e \mathscr{R}^{*} a \leqslant \mathscr{R}^{*} b \mathscr{R}^{*} f
$$

in $T / U$. Thus $f e=e$ and $e \leqslant \mathscr{R} f$ in $S$; thus $a \leqslant_{\mathscr{R}^{*}} b$ in $S$. The dual argument works for $\leqslant \mathscr{L}^{*}$.

Finally, suppose that every $\mathscr{H}^{*}$-class of $T$ contains a regular element. The only element in the $\mathscr{H}^{*}$-class of 0 in $T / U$ is 0 , which is regular. The non-zero $\mathscr{H}^{*}$-classes of $T / U$ are, by the above, $\mathscr{H}^{*}$-classes of $S$ and of $T$. Thus if $H_{a}^{*} \subseteq T \backslash U$, there is an element $b \in H_{a}^{*}$ with $b$ regular in $T$. Now $b=b c b$ for some $c \in T$ and as $U$ is an ideal, we must have $c \in T \backslash U$.

\section{ENDOMORPHISMS}

We recall from Section 2 that a basis algebra is a stable basis algebra if every subalgebra which can be generated by at most rank $A$ elements has a basis, and so is itself a basis algebra. Hereditary basis algebras are stable, and semihereditary basis algebras of finite rank are stable; thus the examples cited at the end of Section 2, namely: free modules of finite rank over a Bezout domain; free $T$-acts on a finite set where $T$ is cancellative and every finitely generated left ideal is principal; and independence algebras are all stable. 
In this section we show that the endomorphism monoid of a stable basis algebra is an abundant monoid with pleasant properties. We start by characterising the relations $\leqslant_{\mathscr{R}}{ }^{*}$ and $\leqslant \mathscr{L}^{*}$, and the corresponding equivalences. We recall from the Introduction that for any algebra $A$, we denote the monoid of endomorphisms by End $A$.

Lemma 4.1. Let $A$ be a relatively free algebra and let $\alpha, \beta \in$ End $A$. Then

$$
\alpha \leqslant \mathscr{R}^{*} \beta \text { if and only if } \operatorname{Ker} \beta \subseteq \operatorname{Ker} \alpha \text {. }
$$

Proof. If $A=\langle\emptyset\rangle$, then End $A=\left\{I_{A}\right\}$ and the result is trivial. Now let $X$ be a non-empty set of free generators for $A$.

If $\operatorname{Ker} \beta \subseteq \operatorname{Ker} \alpha$, then it is well known that $\alpha \leqslant \mathscr{R} \beta$ in $T(A)$, and hence, by Lemma 3.2, $\alpha \leqslant \mathscr{R}^{*} \beta$ in End $A$.

For the converse, suppose that $\alpha \leqslant \mathscr{R}^{*} \beta$ and let $(u, v) \in \operatorname{Ker} \beta$. Since $A$ is relatively free on $X$, there are endomorphisms $\gamma, \delta$ of $A$ with $x \gamma=u$ and $x \delta=v$ for all $x \in X$. If $a \in A$, then $a=t\left(x_{1}, \ldots, x_{n}\right)$ for some term operation $t$ and $x_{1}, \ldots, x_{n} \in X$, and so

$$
\begin{aligned}
a \gamma \beta & =t\left(x_{1}, \ldots, x_{n}\right) \gamma \beta=t\left(x_{1} \gamma \beta, \ldots, x_{n} \gamma \beta\right)=t(u \beta, \ldots, u \beta) \\
& =t(v \beta, \ldots, v \beta)=t\left(x_{1} \delta \beta, \ldots, x_{n} \delta \beta\right)=t\left(x_{1}, \ldots, x_{n}\right) \delta \beta \\
& =a \delta \beta .
\end{aligned}
$$

Thus $\gamma \beta=\delta \beta$ and so $\gamma \alpha=\delta \alpha$ since $\alpha \leqslant \mathscr{R}^{*} \beta$. Now, if $x \in X$, then we have

$$
u \alpha=x \gamma \alpha=x \delta \alpha=v \alpha
$$

so that $(u, v) \in \operatorname{Ker} \alpha$. Hence $\operatorname{Ker} \beta \subseteq \operatorname{Ker} \alpha$.

Corollary 4.2. Let $A$ be a relatively free algebra and let $\alpha, \beta \in$ End $A$. Then

$$
\alpha \mathscr{R}^{*} \beta \quad \text { if and only if } \operatorname{Ker} \alpha=\operatorname{Ker} \beta \text {. }
$$

We use the pure closure of the image of an endomorphism to characterise $\mathscr{L}^{*}$. To do this we need the following result [10, Lemma 6.1].

Lemma 4.3. If $\alpha, \beta$ are endomorphisms of a torsion-free weak independence algebra $A$, then

$$
S_{\alpha, \beta}=\{a \in A: a \alpha=a \beta\}
$$

is a pure subalgebra of $A$.

It is worth recording the following corollary.

Corollary 4.4. If $\alpha$ is an idempotent endomorphism of a torsion-free weak independence algebra $A$, then $\operatorname{Im} \alpha$ is pure in $A$.

Lemma 4.5. Let $A$ be a basis algebra and let $\alpha, \beta \in$ End $A$. Then

$$
\alpha \leqslant \mathscr{L} * \beta \quad \text { if and only if } \mathrm{PC}(\operatorname{Im} \alpha) \subseteq \mathrm{PC}(\operatorname{Im} \beta) .
$$


Proof. Suppose that $\mathrm{PC}(\operatorname{Im} \alpha) \subseteq \mathrm{PC}(\operatorname{Im} \beta)$ and that $\beta \gamma=\beta \delta$ for some endomorphisms $\gamma, \delta$ of $A$. Then $\operatorname{Im} \beta \subseteq S_{\gamma, \delta}$ so that $\mathrm{PC}(\operatorname{Im} \alpha) \subseteq \mathrm{PC}(\operatorname{Im} \beta) \subseteq \mathrm{PC}\left(S_{\gamma, \delta}\right)=S_{\gamma, \delta}$, by Lemma 4.3, so that $\operatorname{Im} \alpha \subseteq S_{\gamma, \delta}$. Hence, for all $a \in A$, we have $a \alpha \gamma=a \alpha \delta$ so that $\alpha \gamma=\alpha \delta$. It follows that $\alpha \leqslant \mathscr{L} * \beta$.

Now suppose that $\alpha \leqslant \mathscr{L}^{*} \beta$. Since $A$ is a basis algebra, $\mathrm{PC}(\operatorname{Im} \beta)$ has a basis $X$ which can be extended to a basis $X \cup Y$ for $A$ where $X \cap Y=\emptyset$. Since a basis is a free generating set, there is an endomorphism $\gamma$ of $A$ with $x \gamma=x$ for all $x \in X$ and $y \gamma \in \operatorname{Im} \beta$ for all $y \in Y$. Note that $\operatorname{Im} \gamma=\langle X\rangle=\mathrm{PC}(\operatorname{Im} \beta)$ and that the restriction of $\gamma$ to $\mathrm{PC}(\operatorname{Im} \beta)$ is the identity map. Hence $\beta \gamma=\beta$ and so $\alpha \gamma=\alpha$ since $\alpha \leqslant \mathscr{L} * \beta$. Thus

$$
\operatorname{Im} \alpha=\operatorname{Im} \alpha \gamma \subseteq \operatorname{Im} \gamma=\mathrm{PC}(\operatorname{Im} \beta),
$$

and it follows that $\mathrm{PC}(\operatorname{Im} \alpha) \subseteq \mathrm{PC}(\operatorname{Im} \beta)$.

Corollary 4.6. Let $A$ be a basis algebra and let $\alpha, \beta \in$ End $A$. Then

$$
\alpha \mathscr{L}^{*} \beta \quad \text { if and only if } \mathrm{PC}(\operatorname{Im} \alpha)=\mathrm{PC}(\operatorname{Im} \beta) \text {. }
$$

Next, we determine the regular elements of End $A$.

Lemma 4.7. Let $\alpha$ be an endomorphism of a basis algebra $A$. Then $\alpha$ is regular if and only if $\operatorname{Im} \alpha=\mathrm{PC}(\operatorname{Im} \alpha)$.

Proof. If $\alpha$ is regular, then $\alpha=\alpha \beta \alpha$ for some endomorphism $\beta$. Now $\beta \alpha$ is idempotent, so that, by Corollary 4.4,

$$
\operatorname{Im} \alpha=\operatorname{Im} \beta \alpha=\mathrm{PC}(\operatorname{Im} \beta \alpha)=\mathrm{PC}(\operatorname{Im} \alpha) .
$$

Conversely, suppose that $\operatorname{Im} \alpha=\mathrm{PC}(\operatorname{Im} \alpha)$, that is, $\operatorname{Im} \alpha$ is pure. Then, since $A$ is a basis algebra, $\operatorname{Im} \alpha$ has a basis $X$ which can be extended to a basis $X \cup Y$ for $A$ where $X \cap Y=\emptyset$. For each $x \in X$, choose $a_{x} \in A$ with $a_{x} \alpha=x$. Since $X \cup Y$ is $A$-free, there is an endomorphism $\beta$ of $A$ with $x \beta=a_{x}$ for all $x \in X$. Then $x \beta \alpha=a_{x} \alpha=x$ for all $x \in X$, and it follows that the restriction of $\beta \alpha$ to $\operatorname{Im} \alpha$ is the identity map. Hence $\alpha \beta \alpha=\alpha$ and $\alpha$ is regular.

It is easy to see that if $A$ is a basis algebra, every member of End $A$ is $\mathscr{L}^{*}$-related to an idempotent, for, if $\alpha$ is an endomorphism, then $\mathrm{PC}(\operatorname{Im} \alpha)$ is a pure subalgebra, and so $\mathrm{PC}(\operatorname{Im} \alpha)$ has a basis $X$ which may be extended to a basis $Z$ for $A$. Since $A$ is a weak independence algebra, there is an endomorphism $\varepsilon$ of $A$ with $x \varepsilon=x$ for all $x \in X$ and $z \varepsilon \in\langle X\rangle$ for all $z \in Z$. Clearly, $\varepsilon$ is idempotent, and $\mathrm{PC}(\operatorname{Im} \varepsilon)=\operatorname{Im} \varepsilon=\mathrm{PC}(\operatorname{Im} \alpha)$, so that, by Lemma 4.6, $\alpha \mathscr{L}^{*} \varepsilon$. Thus End $A$ is abundant if and only if every $\mathscr{R}^{*}$-class contains an idempotent. To ensure this we need an extra condition, as we see in the next theorem. First, we note the following lemma.

Lemma 4.8. Let $\alpha$ be an endomorphism of a stable basis algebra A. Then the subalgebra $\operatorname{Im} \alpha$ is relatively free.

Proof. This is immediate because $A$ has a basis $X$ and $\operatorname{Im} \alpha$ is generated by $X \alpha$. 
Theorem 4.9. Let $A$ be a basis algebra. Then End $A$ is abundant if and only if $A$ is stable. Moreover, when this is the case, every $\mathscr{H}^{*}$-class of End $A$ contains a regular element, End $A$ is $I C$ abundant and $\mathscr{L}^{*} \circ \mathscr{R}^{*}=\mathscr{R}^{*} \circ \mathscr{L}^{*}$.

Proof. Let $A$ have rank $\kappa$ and suppose that End $A$ is abundant. Let $B$ be a subalgebra generated by $\lambda$ elements where $0<\lambda \leqslant \kappa$. Then, since $A$ is relatively free, there is an endomorphism $\alpha$ of $A$ with $\operatorname{Im} \alpha=B$. Since End $A$ is abundant, $\alpha \mathscr{R}^{*} \varepsilon$ for some idempotent endomorphism $\varepsilon$. By Lemma 4.2, $\operatorname{Ker} \alpha=\operatorname{Ker} \varepsilon$ and so $\alpha$ is one-one on $\operatorname{Im} \varepsilon$. Now, by Corollary $4.4, \operatorname{Im} \varepsilon$ is pure, and so it has a basis, $X$ say. By Proposition $2.14, X \alpha$ is directly independent, and since $\varepsilon \alpha=\alpha$, we also have

$$
\langle X \alpha\rangle=\langle X\rangle \alpha=(\operatorname{Im} \varepsilon) \alpha=\operatorname{Im} \varepsilon \alpha=\operatorname{Im} \alpha=B .
$$

Thus $X \alpha$ is a basis for $B$, that is, $B$ is relatively free. Hence $A$ is stable.

Conversely, suppose that $A$ is stable, and let $\alpha \in$ End $A$. Then, by Lemma $4.8, \operatorname{Im} \alpha$ is relatively free. By Lemma 2.18, there is an isomorphism $\theta$ from $\operatorname{Im} \alpha$ to $\operatorname{PC}(\operatorname{Im} \alpha)$. Let $\beta=\alpha \theta$; then $\beta$ is an endomorphism of $A$ with image $\mathrm{PC}(\operatorname{Im} \alpha)$. By Lemma 4.7, $\beta$ is regular since $\mathrm{PC}(\operatorname{Im} \alpha)$ is pure. Also, $\operatorname{Ker} \beta=\operatorname{Ker} \alpha$ since $\theta$ is an isomorphism, so that $\beta \mathscr{R}^{*} \alpha$, by Lemma 4.2. Since $\operatorname{Im} \beta$ is pure, $\mathrm{PC}(\operatorname{Im} \beta)=\operatorname{Im} \beta=\mathrm{PC}(\operatorname{Im} \alpha)$, so that, by Lemma 4.6, $\beta \mathscr{L}^{*} \alpha$. Thus every $\mathscr{H}^{*}$-class of End $A$ contains a regular element, and so, by Lemma 3.3, End $A$ is IC abundant and $\mathscr{L}^{*}$ commutes with $\mathscr{R}^{*}$.

For the rest of the paper, we restrict our attention to stable basis algebras.

By an endomorphism pair of a stable basis algebra $A$, we mean a pair $(\rho, B)$ where $B$ is a pure subalgebra of $A$, and $\rho$ is a congruence on $A$ such that $A / \rho$ is isomorphic $B$.

For such an endomorphism pair, we put

$$
H_{(\rho, B)}^{*}=\{\alpha \in \text { End } A: \operatorname{Ker} \alpha=\rho \text { and } \operatorname{PC}(\operatorname{Im} \alpha)=B\} .
$$

We now characterise the $\mathscr{H}^{*}$-classes of End $A$.

Lemma 4.10. Let $A$ be a stable basis algebra. Then

(1) for an endomorphism pair $(\rho, B)$ of $A$, the set $H_{(\rho, B)}^{*}$ is an $\mathscr{H}^{*}$-class of End $A$;

(2) if $\alpha \in$ End $A$, then $(\operatorname{Ker} \alpha, \mathrm{PC}(\operatorname{Im} \alpha))$ is an endomorphism pair;

(3) if $H^{*}$ is an $\mathscr{H}^{*}$-class of End $A$, then $H^{*}=H_{(\rho, B)}^{*}$ for some endomorphism pair $(\rho, B)$, and the pair $(\rho, B)$ can be taken to be $(\operatorname{Ker} \alpha, \mathrm{PC}(\operatorname{Im} \alpha))$ for any $\alpha$ in $H^{*}$.

Proof. (1) Given the endomorphism pair $(\rho, B)$, let $\nu: A \rightarrow A / \rho$ be the natural map, and $\theta: A / \rho \rightarrow B$ be an isomorphism. Then $\nu \theta: A \rightarrow B$ is an endomorphism of $A$ with image $B$. Moreover, since $\theta$ is an isomorphism it is clear that $\operatorname{Ker} \nu \theta=\rho$. Hence, by Corollaries 4.2 and 4.6,

$$
\begin{aligned}
H_{\nu \theta}^{*} & =\{\alpha \in \text { End } A: \operatorname{Ker} \alpha=\operatorname{Ker} \nu \theta \text { and } \mathrm{PC}(\operatorname{Im} \alpha)=\mathrm{PC}(\operatorname{Im} \nu \theta)\} \\
& =\{\alpha \in \text { End } A: \operatorname{Ker} \alpha=\rho \text { and } \mathrm{PC}(\operatorname{Im} \alpha)=B\} \\
& =H_{(\rho, B)}^{*}
\end{aligned}
$$

so that $H_{(\rho, B)}^{*}$ is an $\mathscr{H}^{*}$-class of End $A$. 
(2) If $\alpha$ is an endomorphism of $A$, then $A / \operatorname{Ker} \alpha \cong \operatorname{Im} \alpha$. Since $A$ is stable, $\operatorname{Im} \alpha$ is relatively free by Lemma 4.8. By Lemma 2.18, $\operatorname{Im} \alpha$ and $\mathrm{PC}(\operatorname{Im} \alpha)$ are isomorphic, and so $A / \operatorname{Ker} \alpha \cong \mathrm{PC}(\operatorname{Im} \alpha)$. Thus $(\operatorname{Ker} \alpha, \mathrm{PC}(\operatorname{Im} \alpha))$ is an endomorphism pair.

(3) Let $\alpha$ be a member of $H^{*}$. Then $(\operatorname{Ker} \alpha, \operatorname{PC}(\operatorname{Im} \alpha))$ is an endomorphism pair, and by Corollaries 4.2 and 4.6,

$$
H_{\alpha}^{*}=\{\beta \in \text { End } A: \operatorname{Ker} \beta=\operatorname{Ker} \alpha \text { and } \mathrm{PC}(\operatorname{Im} \beta)=\mathrm{PC}(\operatorname{Im} \alpha)\}=H_{(\operatorname{Ker} \alpha, \operatorname{PC}(\operatorname{Im} \alpha))}^{*} .
$$

For an endomorphism $\alpha$ of a stable basis algebra $A$, we define the rank of $\alpha$, written $\operatorname{rank} \alpha$, to be the rank of $\operatorname{Im} \alpha$. We are now in a position to characterise the $\mathscr{D}^{*}$-classes of End $A$.

Lemma 4.11. Let $A$ be a stable basis algebra. For $\alpha, \beta \in$ End $A$,

$$
\alpha \mathscr{D}^{*} \beta \quad \text { if and only if } \operatorname{rank} \alpha=\operatorname{rank} \beta .
$$

Proof. If $\alpha \mathscr{D}^{*} \beta$, then, in view of Theorem 4.9, there is an endomorphism $\gamma$ with $\alpha \mathscr{R}^{*} \gamma \mathscr{L}^{*} \beta$. Hence, by Corollaries 4.2 and 4.6, Ker $\alpha=\operatorname{Ker} \gamma$ and $\mathrm{PC}(\operatorname{Im} \gamma)=\mathrm{PC}(\operatorname{Im} \beta)$. But

$$
\operatorname{Im} \alpha \cong A / \operatorname{Ker} \alpha=A / \operatorname{Ker} \gamma \cong \operatorname{Im} \gamma,
$$

so that $\operatorname{rank} \alpha=\operatorname{rank} \gamma$. By Lemma $2.18, \operatorname{Im} \gamma \cong \operatorname{Im} \beta$ so that $\operatorname{rank} \alpha=\operatorname{rank} \beta$ as required.

Conversely, suppose that $\operatorname{rank} \alpha=\operatorname{rank} \beta$. Let $X$ and $Y$ be bases of $\operatorname{Im} \alpha$ and $\operatorname{Im} \beta$ respectively, and let $Z$ be a basis for $\mathrm{PC}(\operatorname{Im} \alpha)$. Since $\operatorname{rank} \operatorname{Im} \beta=\operatorname{rank} \operatorname{Im} \alpha=\operatorname{rank} P C(\operatorname{Im} \alpha)$, the sets $X, Y$ and $Z$ have the same cardinality; let $\theta: Y \rightarrow Z$ be a bijection and put $z_{y}=y \theta$.

For each $y \in Y$, choose $u_{y} \in A$ such that $u_{y} \beta=y$ and put $U=\left\{u_{y}: y \in Y\right\}$. By Proposition 2.14, $U$ is directly independent.

Since $A$ is a weak independence algebra, $Z$ is $A$-free, and hence there is a morphism $\gamma: P C(\operatorname{Im} \alpha) \rightarrow\langle U\rangle$ with $z_{y} \gamma=u_{y}$, and since the restriction of $\gamma$ to $Z$ is one-one, $\gamma$ is itself one-one by Lemma 2.12. Hence $\gamma$ maps $X$ bijectively onto $X \gamma$.

Similarly, since $\beta$ maps $U$ bijectively onto $Y$, the restriction of $\beta$ to $\langle U\rangle$ is one-one, and, in particular, $\beta$ is one-one on $\langle X \gamma\rangle$. Hence, letting $\delta$ be the self-map of $A$ defined by

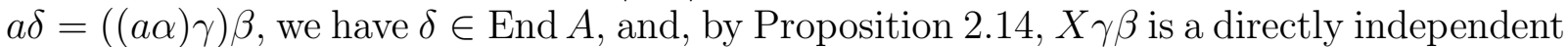
subset of $\operatorname{Im} \delta$, and hence of $\operatorname{Im} \beta$.

We claim that $X \gamma \beta$ is a maximal directly independent subset of $\operatorname{Im} \beta$. Now $X$ is a maximal directly independent subset of $\operatorname{Im} \alpha$ so that, by Lemma 2.2, $\mathrm{PC}(X)=\operatorname{PC}(\operatorname{Im} \alpha)$, and hence $Z \prec X$. Also $\gamma$ is one-one, and the restriction of $\beta$ to $\langle U\rangle$ is one-one, so by Lemma 2.16, $Z \gamma \prec X \gamma$ and $Z \gamma \beta \prec X \gamma \beta$, that is, $Y \prec X \gamma \beta$. Since $Y$ is maximal directly independent in $\operatorname{Im} \beta$, it follows from condition $(\mathrm{T})$ that $\mathrm{PC}(\operatorname{Im} \beta)=\mathrm{PC}(Y) \subseteq$ $\mathrm{PC}(X \gamma \beta) \subseteq \mathrm{PC}(\operatorname{Im} \beta)$, so that $\mathrm{PC}(X \gamma \beta)=\mathrm{PC}(\operatorname{Im} \beta)$. But $X \gamma \beta \subseteq \operatorname{Im} \delta \subseteq \operatorname{Im} \beta$, and so $\mathrm{PC}(\operatorname{Im} \delta)=\mathrm{PC}(\operatorname{Im} \beta)$ since $\mathrm{PC}$ is a closure operator. Therefore, by Corollary $4.6, \delta \mathscr{L}^{*} \beta$.

Clearly, Ker $\alpha \subseteq \operatorname{Ker} \delta$. Suppose that $a, b \in A$ and $a \delta=b \delta$. Since $\beta$ is one-one on $\langle U\rangle$ and $a \alpha \gamma, b \alpha \gamma \in\langle U\rangle$, we have $a \alpha \gamma=b \alpha \gamma$. Hence $a \alpha=b \alpha$ since $\gamma$ is an isomorphism. Thus $(a, b) \in \operatorname{Ker} \alpha$ so that $\operatorname{Ker} \alpha=\operatorname{Ker} \delta$, and, by Corollary $4.2, \alpha \mathscr{R}^{*} \delta$.

Thus $\alpha \mathscr{D}^{*} \beta$ as required. 
We conclude this section by locating the idempotents of End $A$.

Lemma 4.12. Let $(\rho, B)$ be an endomorphism pair of a stable basis algebra $A$. Then the $\mathscr{H}^{*}$-class $H_{(\rho, B)}^{*}$ contains an idempotent if and only if every $\rho$-class contains a unique element of $B$.

Proof. Suppose that $\varepsilon=\varepsilon^{2} \in H_{(\rho, B)}^{*}$. Then $\operatorname{Ker} \varepsilon=\rho$, and since, by Corollary 4.4, $\operatorname{Im} \varepsilon$ is pure in $A$, we have $\operatorname{Im} \varepsilon=B$. For any $a \in A$, we have $a \varepsilon \in B$ and $a \varepsilon^{2}=a \varepsilon$ so that $a \varepsilon \in a \rho$, and every $\rho$-class contains an element of $B$.

If $u, v \in B$ and $u \rho v$, then $u=u \varepsilon=v \varepsilon=v$, and so there is only one element of $B$ in each $\rho$-class.

For the converse, define $\mu: A \rightarrow A$ by $a \mu=b_{a}$ where $a \rho b_{a}$ and $b_{a} \in B$. Certainly, $\mu$ is well defined. If $t$ is an $n$-ary basic operation of $A$, and $a_{1}, \ldots, a_{n} \in A$, then $t\left(b_{a_{1}}, \ldots, b_{a_{n}}\right) \in B$ and, since $\rho$ is a congruence, $t\left(a_{1}, \ldots, a_{n}\right) \rho t\left(b_{a_{1}}, \ldots, b_{a_{n}}\right)$. Hence

$$
t\left(a_{1}, \ldots, a_{n}\right) \mu=t\left(b_{a_{1}}, \ldots, b_{a_{n}}\right)=t\left(a_{1} \mu \ldots, a_{n} \mu\right),
$$

and $\mu$ is an endomorphism. Clearly $\operatorname{Im} \mu=B$, and the restriction of $\mu$ to $B$ is the identity, so $\mu$ is idempotent. Clearly, also Ker $\mu=\rho$ so that $\mu \in H_{(\rho, B)}^{*}$.

\section{LEFT ORDERS}

In this section we give the background on the theory of left orders necessary for the remainder of the paper. For any element $q$ of a semigroup $Q, q^{\sharp}$ denotes the inverse of $q$ in a subgroup of $Q$. Use of the notation $q^{\sharp}$ implies that $q$ lies in a subgroup of $Q ; q^{\sharp}$ is then uniquely determined as the (group) inverse of $q$ in the subgroup $H_{q}$ of $Q$. The union of subgroups of $Q$ is denoted by $\mathcal{H}(Q)$.

An element $a$ of a semigroup $S$ is square cancellable if $a \mathscr{H}^{*} a^{2}$. If $a$ lies in a subgroup of an oversemigroup of $S$, then $a$ and $a^{2}$ are $\mathscr{H}$-related in the oversemigroup (see [18]), and hence $a$ is square cancellable (in $S$ ). In view of Lemma 3.2, being square cancellable is a strong necessary condition for an element of $S$ to lie in a subgroup of an oversemigroup. We denote by $\mathcal{S}(S)$ the set of square cancellable elements of $S$.

We say that a subsemigroup $S$ of a semigroup $Q$ is a left order in $Q$ if every element of $Q$ can be written as $a^{\sharp} b$ for some $a, b \in S$ and if, in addition, every square cancellable element of $S$ lies in a subgroup of $Q$.

It is easy to see that if $S$ is a left order in $Q$, then any element of $Q$ may be written as $a^{\sharp} b$ where $a, b \in S, b \leqslant \mathscr{R} a$ in $Q$ and $a^{\sharp} b \mathscr{L} b$ in $Q$. Thus $S$ intersects every $\mathscr{L}$-class of $Q$. If moreover $S$ intersects every $\mathscr{H}$-class of $Q$, then $S$ inherits more of the structure of $Q$. One way of ensuring this is by using the concept of straightness.

Let $S$ be a left order in $Q$. We say that $S$ is straight in $Q$ if every element of $Q$ can be written as $a^{\sharp} b$ where $a, b \in S$ and $a \mathscr{R} b$ in $Q$. For such elements $a$ and $b$ it is easy to see that $a^{\sharp} b \mathscr{H} b$ in $Q$, so that $S$ intersects every $\mathscr{H}$-class of $Q$. Note also that $Q$ must be regular.

The main result of [15] is a complete characterisation of those semigroups that are straight left orders. This is specialised in [16] to abundant straight left orders. The 
approach of [15] is via embeddable *-pairs, which are pairs of preorders whose properties reflect those of $\leqslant \mathscr{L}$ and $\leqslant \mathscr{R}$ on a regular semigroup.

An ordered pair $\mathcal{P}=\left(\leqslant_{l}, \leqslant_{r}\right)$ of preorders on a semigroup $S$ is a $*$-pair if $\leqslant_{l}$ is right compatible with multiplication, $\leqslant_{r}$ is left compatible, $\leqslant_{l} \subseteq \leqslant_{\mathscr{L}^{*}}$ and $\leqslant_{r} \subseteq \leqslant_{\mathscr{R}^{*}}$. Clearly $\mathcal{P}^{*}=\left(\leqslant \mathscr{L}^{*}, \leqslant \mathscr{R}^{*}\right)$ is a $*$-pair for any semigroup $S$. As a consequence of Lemma 3.2 we have the following.

Corollary 5.1. Let $S$ be a subsemigroup of $Q$. Then

$$
\left(\leqslant_{\mathcal{L}^{Q}} \cap(S \times S), \leqslant_{\mathcal{R}^{Q}} \cap(S \times S)\right)
$$

is $a *$-pair for $S$.

The *-pair of the corollary is called the *-pair for $S$ induced by $Q$. In particular, the corollary applies when $S$ is a left order in $Q$. In this case, if $Q$ induces $\mathcal{P}^{*}$ we say that $S$ is fully stratified in $Q$.

We caution the reader that the labelling of the conditions in the following result differs from that used in [15] and [16]. Recall that a subset $H$ of a semigroup $S$ satisfies the left Ore condition if for any $a, b \in H$ there exist $c, d \in H$ with $c a=d b$.

Theorem 5.2. [16, Proposition 4.1] Let $S$ be an $I C$ abundant semigroup. Then $S$ is a fully stratified straight left order in a semigroup $Q$ inducing $\mathcal{P}^{*}$ if and only if $S$ satisfies the following conditions and the left-right dual of (2):

(1) $\mathscr{L}^{*} \circ \mathscr{R}^{*}=\mathscr{R}^{*} \circ \mathscr{L}^{*}$;

(2) for all $a \in \mathcal{S}(S)$ and $b, c \in S$, if $b, c \leqslant \mathscr{R}^{*} a$ and $a b=a c$, then $b=c$;

(3) for all $a \in \mathcal{S}(S)$ and $b, c \in S$, if $b, c \leqslant \mathscr{R}^{*} a$ and $a b \mathscr{R}^{*} a c$, then $b \mathscr{R}^{*} c$;

(4) for all $a \in \mathcal{S}(S)$, $H_{a}^{*}$ satisfies the left Ore condition.

Moreover, with the notation of the theorem, we have the following result by Corollary 4.3 of [13].

Corollary 5.3. The semigroup $Q$ is uniquely determined by $S$.

\section{LeFt ORDERS AND EndOMORPHISMS}

In this section, we examine further the $\mathscr{H}^{*}$-classes of the endomorphism monoid of a stable basis algebra $A$. Our aim is to show that the subsemigroup End $A$ of End $A$ consisting of all endomorphisms of finite rank is a fully stratified straight left order in a regular semigroup. That $\operatorname{End}_{f} A$ is a subsemigroup (indeed, an ideal) of End $A$ is a consequence of the following lemma.

Lemma 6.1. If $\alpha, \beta$ are endomorphisms of a stable basis algebra $A$, then rank $\alpha \beta \leqslant$ $\min \{\operatorname{rank} \alpha, \operatorname{rank} \beta\}$.

Proof. Since $\operatorname{Im} \alpha \beta \subseteq \operatorname{Im} \beta$, we have $\operatorname{rank} \alpha \beta \leqslant \operatorname{rank} \beta$. By Corollary 4.8, $\operatorname{Im} \alpha$ has a basis $X$, so that $\operatorname{Im} \alpha=\langle X\rangle$ and

$$
\operatorname{Im} \alpha \beta=(\operatorname{Im} \alpha) \beta=\langle X\rangle \beta=\langle X \beta\rangle .
$$


Hence by Proposition 2.6,

$$
\operatorname{rank} \alpha \beta=\operatorname{rank} \operatorname{Im} \alpha \beta=\operatorname{rank}\langle X \beta\rangle \leqslant|X \beta| \leqslant|X|=\operatorname{rank} \alpha .
$$

For a cardinal $\mu$ and a stable basis algebra $A$, it follows from Lemmas 4.11 and 6.1 that

$$
T_{\mu}=\{\alpha \in \operatorname{End} A: \operatorname{rank} \alpha<\mu\}
$$

is a $*$-ideal of End $A$. We denote $T_{\aleph_{0}}$ by $\operatorname{End}_{f} A$.

Corollary 6.2. Let $A$ be a stable basis algebra. Then $\operatorname{End}_{f} A$ is a *-ideal of End $A$.

To describe the relations $\leqslant \mathscr{R}^{*}$ and $\leqslant \mathscr{L}^{*}$ on $\operatorname{End}_{f} A$ we employ Lemma 3.5 together with the results of Section 4.

Corollary 6.3. Let $A$ be a stable basis algebra. Then for any $\alpha, \beta \in \operatorname{End}_{f} A$,

$$
\alpha \leqslant \mathscr{R}^{*} \beta \text { in } \operatorname{End}_{f} A \text { if and only if } \operatorname{Ker} \beta \subseteq \operatorname{Ker} \alpha
$$

and

$$
\alpha \leqslant \mathscr{L}^{*} \beta \text { in } \operatorname{End}_{f} A \text { if and only if } \mathrm{PC}(\operatorname{Im} \alpha) \subseteq \mathrm{PC}(\operatorname{Im} \beta) .
$$

In $\operatorname{End}_{f} A, \mathscr{D}^{*}=\mathscr{L}^{*} \circ \mathscr{R}^{*}=\mathscr{R}^{*} \circ \mathscr{L}^{*}$, every $\mathscr{H}^{*}$-class contains a regular element and End $_{f} A$ is IC abundant.

In particular, we note that $\operatorname{End}_{f} A$ satisfies condition (1) of Theorem 5.2. We now consider the square cancellable elements in End $A$.

Lemma 6.4. Let $A$ be a stable basis algebra. If $\alpha$ is a square cancellable endomorphism of $A$, then the restriction of $\alpha$ to $P C(\operatorname{Im} \alpha)$ is one-one.

Proof. By Corollaries 4.6 and 4.2, we have $\mathrm{PC}(\operatorname{Im} \alpha)=\mathrm{PC}\left(\operatorname{Im} \alpha^{2}\right)$ and $\operatorname{Ker} \alpha=\operatorname{Ker} \alpha^{2}$. Thus $\alpha$ is one-one on $\operatorname{Im} \alpha$.

Let $x, y \in \mathrm{PC}(\operatorname{Im} \alpha)$ and suppose that $x \alpha=y \alpha$. If $x \in\langle\emptyset\rangle$, then $x \in \operatorname{Im} \alpha$ so that either $x=y$ or $y \notin \operatorname{Im} \alpha$. In the latter case, there is a term operation $t$ such that $t(y) \in \operatorname{Im} \alpha \backslash\langle\emptyset\rangle$, and thus

$$
t(y) \alpha=t(y \alpha)=t(x \alpha)=t(x) \alpha
$$

so that $t(y)=t(x) \in\langle\emptyset\rangle$, a contradiction.

Therefore, we can assume that $x, y$ are not in the constant subalgebra, so that there is a term operation $u$ with $u(y) \in \operatorname{Im} \alpha \backslash\langle\emptyset\rangle$. Then $u(x) \alpha=u(y) \alpha$ and so $u(x) \notin\langle\emptyset\rangle$. Since $u(x) \prec\{x\} \prec \operatorname{Im} \alpha$, condition (T) of Lemma 2.5 gives that $u(x) \prec \operatorname{Im} \alpha$, so there is a term operation $s$ such that $s(u(x)) \in \operatorname{Im} \alpha \backslash\langle\emptyset\rangle$. Certainly, $s(u(y)) \in \operatorname{Im} \alpha$ and since $(s u(x)) \alpha=(s u(y)) \alpha$ we have $s u(x)=s u(y)$. By torsion-freeness, $x=y$ as required.

We specialise now to elements of $\operatorname{End}_{f} A$.

Lemma 6.5. Let $A$ be a stable basis algebra. If $\alpha$ is square cancellable element of $\operatorname{End}_{f} A$, then every element of $H_{\alpha}^{*}$ is square cancellable. 
Proof. Let $\gamma \in$ End $A$ with $\gamma \mathscr{H}^{*} \alpha$. Then, since $\mathscr{L}^{*}$ is a right congruence, we have $\gamma \alpha \mathscr{L}^{*} \alpha^{2}$ so that $\gamma \alpha \mathscr{L}^{*} \alpha$. By Corollary 4.6, we have $\mathrm{PC}(\operatorname{Im} \alpha)=\mathrm{PC}(\operatorname{Im} \gamma)$, and, by Lemma $6.4, \alpha$ is one-one on $\mathrm{PC}(\operatorname{Im} \alpha)$. Hence, if $a, b \in A$ are such that $(a, b) \in \operatorname{Ker} \gamma \alpha$, then $a \gamma \alpha=b \gamma \alpha$ so that $a \gamma=b \gamma$, that is, $(a, b) \in \operatorname{Ker} \gamma$, and we have $\operatorname{Ker} \gamma \alpha \subseteq \operatorname{Ker} \gamma$. The opposite inclusion is obvious, and so, by Corollary $4.2, \gamma \alpha \mathscr{R}^{*} \gamma$. Thus $\gamma \alpha \in H_{\alpha}^{*}$.

Now $\operatorname{Ker} \gamma=\operatorname{Ker} \alpha$ and $\alpha$ is one-one on $\operatorname{PC}(\operatorname{Im} \alpha)$, so that $\gamma$ is one-one on $\operatorname{PC}(\operatorname{Im} \alpha)$. Let $X$ be a basis of $\operatorname{Im} \alpha$. Then, since $X$ is finite and

$$
|X|=|X \gamma|=\operatorname{rank} \operatorname{Im} \gamma,
$$

we have $\mathrm{PC}(X \gamma)=\mathrm{PC}(\operatorname{Im} \gamma)$, so that

$$
\mathrm{PC}(\operatorname{Im} \alpha \gamma) \supseteq \mathrm{PC}(X \gamma)=\mathrm{PC}(\operatorname{Im} \gamma)=\mathrm{PC}(\operatorname{Im} \alpha) .
$$

Since $\operatorname{Im} \alpha \gamma \subseteq \operatorname{Im} \gamma \subseteq \mathrm{PC}(\operatorname{Im} \alpha)$ we have $\mathrm{PC}(\operatorname{Im} \alpha \gamma)=\mathrm{PC}(\operatorname{Im} \alpha)$, so that, by Corollary 4.6, $\alpha \gamma \mathscr{L}^{*} \alpha$. Now $\alpha \gamma \mathscr{R}^{*} \alpha$ follows from $\gamma \mathscr{R}^{*} \alpha$ and $\alpha \mathscr{R}^{*} \alpha^{2}$ since $\mathscr{R}^{*}$ is a left congruence, and so $\alpha \gamma \in H_{\alpha}^{*}$.

Now, from $\gamma \mathscr{L}^{*} \alpha$ we have $\gamma^{2} \mathscr{L}^{*} \alpha \gamma \mathscr{L}^{*} \gamma$, and similarly, $\gamma^{2} \mathscr{R}^{*} \gamma$, so that $\gamma$ is square cancellable.

Corollary 6.6. Let $A$ be a stable basis algebra. If $\alpha$ is square cancellable element of End $_{f} A$, then $H_{\alpha}^{*}$ is a right cancellative subsemigroup of $\operatorname{End}_{f} A$.

Proof. Let $\beta, \gamma \in H_{\alpha}^{*}$. Then, by Lemma $6.5, \beta^{2}, \gamma^{2} \in H_{\alpha}^{*}$. From $\beta \mathscr{L}^{*} \gamma$ we get $\beta^{2} \mathscr{L}^{*} \gamma \beta$, and from $\beta \mathscr{R}^{*} \gamma$ we get $\gamma \beta \mathscr{R}^{*} \gamma^{2}$. It follows that $\gamma \beta \in H_{\alpha}^{*}$ so that $H_{\alpha}^{*}$ is a subsemigroup of $\operatorname{End}_{f} A$.

To see that it is right cancellative, let $\beta, \gamma, \delta \in H_{\alpha}^{*}$ be such that $\beta \delta=\gamma \delta$. Then, for any $a \in A$, we have $a \beta \delta=a \gamma \delta$. Now $\mathrm{PC}(\operatorname{Im} \beta)=\mathrm{PC}(\operatorname{Im} \gamma)=\mathrm{PC}(\operatorname{Im} \delta)$ by Lemma 4.6, so that $a \beta, a \gamma \in \mathrm{PC}(\operatorname{Im} \delta)$. Also, $\delta$ is square cancellable by Lemma 6.5 , and so $\delta$ is one-one on $\mathrm{PC}(\operatorname{Im} \delta)$ by Lemma 6.4. Hence, $a \beta=a \gamma$ so that $\beta=\gamma$.

The next lemma shows that $\operatorname{End}_{f} A$ satisfies condition (4) of Theorem 5.2.

Lemma 6.7. Let $A$ be a stable basis algebra and let $H^{*}$ be an $\mathscr{H}^{*}$-class of a square cancellable element of $\operatorname{End}_{f} A$. Then the subsemigroup $H^{*}$ is left Ore.

Proof. Let $\alpha, \beta \in H^{*}$ with $\operatorname{rank} \alpha=n$. By Corollary 4.6,

$$
\mathrm{PC}\left(\operatorname{Im} \alpha^{2}\right)=\mathrm{PC}(\operatorname{Im} \alpha)=\mathrm{PC}(\operatorname{Im} \beta)=\mathrm{PC}\left(\operatorname{Im} \beta^{2}\right),
$$

so that by Corollary 2.9,

$$
\mathrm{PC}\left(\operatorname{Im} \alpha^{2} \cap \operatorname{Im} \beta^{2}\right)=\mathrm{PC}(\operatorname{Im} \alpha) .
$$

Let $U$ be a maximal directly independent subset of $\operatorname{Im} \alpha^{2} \cap \operatorname{Im} \beta^{2}$. Then $U$ is also maximal in $\mathrm{PC}(\operatorname{Im} \alpha)$.

Since $U \subseteq \operatorname{Im} \alpha^{2}$ we can choose, for each $u \in U$, an element $a_{u} \in \operatorname{Im} \alpha$ such that $a_{u} \alpha=u$. Put $X=\left\{a_{u}: u \in U\right\}$. By Proposition 2.14, $X$ is directly independent and as $|X|=\operatorname{rank} \alpha, X$ is a maximal directly independent subset of $\operatorname{Im} \alpha$.

Let $\varepsilon$ be an idempotent endomorphism with $\varepsilon \mathscr{R}^{*} \alpha$. By Corollary $4.4, \operatorname{Im} \varepsilon$ is a pure subalgebra, so that as $A$ is a basis algebra, $\operatorname{Im} \varepsilon$ has basis $Z$ with $|Z|=n$ by Lemma 4.11 
since $\varepsilon \mathscr{D}^{*} \alpha$. Let $\tau: U \rightarrow Z$ be a bijection and put $z_{u}=u \tau$. From Lemma 2.8 there is an isomorphism $\gamma:\langle Z\rangle=\operatorname{Im} \varepsilon \rightarrow\langle X\rangle$ such that $z_{u} \gamma=a_{u}$. Note that $\varepsilon \gamma \in$ End $A$ and as $\gamma$ is one-one, $\operatorname{Ker} \varepsilon \gamma=\operatorname{Ker} \varepsilon$ so that by Corollary 4.2 ,

$$
\varepsilon \gamma \mathscr{R}^{*} \varepsilon \mathscr{R}^{*} \alpha .
$$

We also have

$$
X \subseteq \operatorname{Im} \varepsilon \gamma=\langle Z\rangle \gamma=\langle Z \gamma\rangle=\left\langle\left\{a_{u}: u \in U\right\}\right\rangle \subseteq \operatorname{Im} \alpha
$$

and as $\mathrm{PC}(X)=\mathrm{PC}(\operatorname{Im} \alpha)$, we have $\mathrm{PC}(\operatorname{Im} \alpha)=\mathrm{PC}(\operatorname{Im} \varepsilon \gamma)$ so that $\alpha \mathscr{L}^{*} \varepsilon \gamma$. Hence $\varepsilon \gamma \in H^{*}$.

Note that $z_{u} \varepsilon \gamma \alpha=z_{u} \gamma \alpha=a_{u} \alpha=u$.

Similarly, there is an endomorphism $\varepsilon \delta$ in $H^{*}$ such that $z_{u} \varepsilon \delta \beta=u$. Thus $\varepsilon \gamma \alpha$ and $\varepsilon \delta \beta$ agree on $Z$, and hence on $\operatorname{Im} \varepsilon$. It follows that $\varepsilon \gamma \alpha=\varepsilon \delta \beta$, and so $H^{*}$ is left Ore, as required.

Corollary 6.8. Let $\varepsilon$ be an idempotent endomorphism of finite rank of a stable basis algebra A. Then $H_{\varepsilon}^{*}$ is a cancellative left Ore subsemigroup of $\operatorname{End}_{f} A$ with identity $\varepsilon$.

Proof. By Lemma 1.12 of [6], such an $\mathscr{H}^{*}$-class is a cancellative monoid (under composition). As $\varepsilon$ is square cancellable, the left Ore condition follows from Lemma 6.7.

We now prove that the left-right dual of condition (2) of Theorem 5.2 holds.

Lemma 6.9. Let $\alpha, \beta, \gamma$ be endomorphisms of a stable basis algebra $A$ with $\alpha$ square cancellable and $\beta, \gamma \leqslant \mathscr{L}^{*} \alpha$. If $\beta \alpha=\gamma \alpha$, then $\beta=\gamma$.

Proof. Let $a \in A$. Then $a \beta \alpha=a \gamma \alpha$. Since $\beta, \gamma \leqslant \mathscr{L}^{*} \alpha$ we have $\mathrm{PC}(\operatorname{Im} \beta) \subseteq \mathrm{PC}(\operatorname{Im} \alpha)$ and $\mathrm{PC}(\operatorname{Im} \gamma) \subseteq \mathrm{PC}(\operatorname{Im} \alpha)$ by Lemma 4.5. Now $\alpha$ is one-one on $\mathrm{PC}(\operatorname{Im} \alpha)$ by Lemma 6.4 , and so $a \beta=a \gamma$ giving $\beta=\gamma$.

To show that $\operatorname{End}_{f} A$ is a fully stratified straight left order we have to verify the remaining criteria of Theorem 5.2, namely, conditions (2) and (3). We start with condition (2).

Lemma 6.10. Let $A$ be a stable basis algebra and let $\alpha, \beta, \gamma \in \operatorname{End}_{f} A$ be such that $\alpha$ is square cancellable, $\beta, \gamma \leqslant \mathscr{R}^{*} \alpha$ and $\alpha \beta=\alpha \gamma$. Then $\beta=\gamma$.

Proof. First, we show that if $\varepsilon$ is an idempotent endomorphism with $\varepsilon \mathscr{R}^{*} \alpha$, then $\alpha \varepsilon \mathscr{L}^{*} \varepsilon$. By Corollary $4.2, \operatorname{Ker} \varepsilon=\operatorname{Ker} \alpha$ and, since $\alpha$ is square cancellable, $\operatorname{Ker} \alpha=\operatorname{Ker} \alpha^{2}$. Hence $\alpha$ is one-one on $\operatorname{Im} \alpha$, and $\operatorname{so} \varepsilon$ is also one-one on $\operatorname{Im} \alpha$. Let $X$ be a basis of $\operatorname{Im} \alpha$. Then, $|X|=|X \varepsilon|$, and, by Lemma 2.14, $X \varepsilon$ is directly independent. Now rank $\alpha=\operatorname{rank} \varepsilon$ since $\alpha$ and $\varepsilon$ are $\mathscr{D}^{*}$-related, and so, since $\varepsilon$ has finite rank, $|X \varepsilon|=\operatorname{rank} \varepsilon$ and $X \varepsilon$ must be a maximal directly independent subset of $\operatorname{Im} \varepsilon$. Hence $\operatorname{Im} \varepsilon=\operatorname{PC}(X \varepsilon)$. Now $X \varepsilon \subseteq \operatorname{Im} \alpha \varepsilon \subseteq \operatorname{Im} \varepsilon$ so that $\mathrm{PC}(\operatorname{Im} \alpha \varepsilon)=\operatorname{Im} \varepsilon$. Thus $\alpha \varepsilon \mathscr{L}^{*} \varepsilon$.

Now $\beta, \gamma \leqslant \mathscr{R}^{*} \varepsilon$ so that $\varepsilon \beta=\beta$ and $\varepsilon \gamma=\gamma$. Now we have

$$
(\alpha \varepsilon) \beta=\alpha(\varepsilon \beta)=\alpha \beta=\alpha \gamma=\alpha(\varepsilon \gamma)=(\alpha \varepsilon) \gamma .
$$

But $\alpha \varepsilon \mathscr{L}^{*} \varepsilon$, and so $\varepsilon \beta=\varepsilon \gamma$, that is, $\beta=\gamma$. 
Finally, we show that condition (3) holds.

Lemma 6.11. Let $A$ be a stable basis algebra and let $\alpha, \beta, \gamma \in \operatorname{End}_{f} A$ be such that $\alpha$ is square cancellable, $\beta, \gamma \leqslant \mathscr{R}^{*} \alpha$ and $\alpha \beta \mathscr{R}^{*} \alpha \gamma$. Then $\beta \mathscr{R}^{*} \gamma$.

Proof. As $\alpha \beta \mathscr{R}^{*} \alpha \gamma$, we have $\operatorname{Ker} \alpha \beta=\operatorname{Ker} \alpha \gamma$ by Corollary 4.2.

As in the proof of Lemma 6.10, if $\varepsilon$ is an idempotent in the $\mathscr{R}^{*}$-class of $\alpha$, then $\varepsilon \beta=$ $\beta, \varepsilon \gamma=\gamma$ and $\alpha \varepsilon \mathscr{L}^{*} \varepsilon$. From the latter, we have $\mathrm{PC}(\operatorname{Im} \alpha \varepsilon)=\mathrm{PC}(\operatorname{Im} \varepsilon)=\operatorname{Im} \varepsilon$ by Corollaries 4.6 and 4.4 .

Let $x, y \in \operatorname{Im} \varepsilon$ and suppose that $x \beta=y \beta$. If $x, y \in\langle\emptyset\rangle$, then $x=x \beta=y \beta=y$ so that $x \gamma=y \gamma$.

If $x \in\langle\emptyset\rangle$ but $y \notin\langle\emptyset\rangle$, then $x \beta=x=x \alpha=x \gamma$ and $t(y) \in \operatorname{Im} \alpha \varepsilon \backslash\langle\emptyset\rangle$ for some unary term operation $t$. Let $t(y)=a \alpha \varepsilon$. Then

$$
t(x) \alpha \beta=t(x \alpha \beta)=t(x \beta)=t(y \beta)=t(y) \beta=a \alpha \varepsilon \beta=a \alpha \beta,
$$

and consequently,

$$
t(x)=t(x) \alpha \gamma=a \alpha \gamma=a \alpha \varepsilon \gamma=t(y) \gamma=t(y \gamma) .
$$

Hence, since $A$ is torsion-free, $y \gamma=x=x \gamma$.

Finally, suppose that neither $x$ nor $y$ is a constant. As above, $t(y) \in \operatorname{Im} \alpha \varepsilon \backslash\langle\emptyset\rangle$ for some unary term operation $t$. By Proposition 2.10, $t(x) \notin\langle\emptyset\rangle$ and so, as in Lemma 6.4, there is a unary term operation $u$ such that $u t(x) \in \operatorname{Im} \alpha \varepsilon \backslash\langle\emptyset\rangle$. It follows that $u t(y) \in \operatorname{Im} \alpha \varepsilon \backslash\langle\emptyset\rangle$. Let $u t(x)=a \alpha \varepsilon$ and $u t(y)=b \alpha \varepsilon$. Then

$$
a \alpha \beta=a \alpha \varepsilon \beta=u t(x) \beta=u t(x \beta)=u t(y \beta)=u t(y) \beta=b \alpha \varepsilon \beta=b \alpha \beta,
$$

so that $a \alpha \gamma=b \alpha \gamma$ and hence

$$
u t(x \gamma)=u t(x) \gamma=a \alpha \varepsilon \gamma=a \alpha \gamma=b \alpha \gamma=b \alpha \varepsilon \gamma=u t(y) \gamma=u t(y \gamma) .
$$

Thus, again by torsion-freeness, $x \gamma=y \gamma$.

It follows that $\operatorname{Ker} \varepsilon \beta \subseteq \operatorname{Ker} \varepsilon \gamma$, and, similarly, we have the opposite inclusion. But $\varepsilon \beta=\beta$ and $\varepsilon \gamma=\gamma$ so that $\operatorname{Ker} \beta=\operatorname{Ker} \gamma$, and $\beta \mathscr{R}^{*} \gamma$ by Corollary 4.2.

Now, in view of the preceding results, we can apply Theorem 5.2 to obtain the following theorem.

Theorem 6.12. Let $A$ be a stable basis algebra. Then $\operatorname{End}_{f} A$ is a fully stratified straight left order in a regular semigroup.

We conjecture that in many cases, the regular semigroup in our theorem above is the monoid of endomorphisms of finite rank of an independence algebra. As evidence for this, consider a free (left) module $F$ of finite rank $n$ over a Bezout domain $R$. Then $R$ is left and right Ore, and so it has a division ring $D$ of (left and right) quotients. Also, $V=D \otimes F$ is a left vector space over $D$, and $F$ embeds in $V$. Now $\operatorname{End}_{R}(F) \cong \mathrm{M}_{n}(R)$ and $\operatorname{End}_{D}(V) \cong \mathrm{M}_{n}(D)$, and it is well known that the matrix ring $\mathrm{M}_{n}(D)$ is a ring of (left and right) quotients of $\mathrm{M}_{n}(R)$. By Corollary 3.6 and Lemma 5.1 of [8] and Corollary 3.11 of [9], $\mathrm{M}_{n}(R)$ is a fully stratified straight order in $\mathrm{M}_{n}(D)$ (in the semigroup sense). Hence, by uniqueness, the regular semigroup of the theorem is $\mathrm{M}_{n}(D)$. 


\section{7. *-IDEALS}

For a cardinal $\mu$ and a stable basis algebra $A$ we have already remarked that

$$
T_{\mu}=\{\alpha \in \text { End } A: \operatorname{rank} \alpha<\mu\}
$$

is a $*$-ideal of End $A$. Lemma 3.5 together with Lemmas 4.1 and 4.5 describe the relations $\leqslant_{\mathscr{R}^{*}}$ and $\leqslant \mathscr{L}^{*}$ on $T_{\mu}$. Similarly, from Theorem 4.9 , we also have that $T_{\mu}$ is IC abundant and $\mathscr{R}^{*} \circ \mathscr{L}^{*}=\mathscr{L}^{*} \circ \mathscr{R}^{*}$ in $T_{\mu}$. If $\mu^{+}$is the successor of $\mu$, notice that

$$
T_{\mu^{+}} \backslash T_{\mu}=\{\alpha \in \text { End } A: \operatorname{rank} \alpha=\mu\}
$$

which is a $\mathscr{D}^{*}$-class of End $A$ by Lemma 4.11. Again by Lemma 3.5, the relations $\leqslant \mathscr{R}^{*}$ and $\leqslant \mathscr{L}^{*}$ on $T_{\mu^{+}} / T_{\mu}$ have a description corresponding to that for End $A$. Moreover, $T_{\mu^{+}} / T_{\mu}$ is IC abundant, every $\mathscr{H}^{*}$-class contains a regular element, $\mathscr{R}^{*} \circ \mathscr{L}^{*}=\mathscr{L}^{*} \circ \mathscr{R}^{*}$ and the non-zero elements form a $\mathscr{D}^{*}$-class.

Before studying these Rees quotients for finite $\kappa$ in greater detail, we point out that the $T_{\kappa}$ are the only $*$-ideals of End $A$.

Proposition 7.1. Let $A$ be a stable basis algebra. If $I$ is a $*$-ideal of End $A$, then $I=T_{\mu}$ for some cardinal $\mu$.

Proof. Let $\alpha \in I$ and let $\beta \in$ End $A$ be such that $\operatorname{rank} \beta \leqslant \operatorname{rank} \alpha$. If $\operatorname{rank} \beta=\operatorname{rank} \alpha$, then by Lemma $4.11, \beta \mathscr{D}^{*} \alpha$, and since $I$ is a $*$-ideal, it follows that $\beta \in I$.

If $\operatorname{rank} \beta<\operatorname{rank} \alpha$, let $Z$ be a basis for $A$, let $X$ be a subset of $Z$ with cardinality rank $\alpha$ and let $Y$ be a subset of $X$ of cardinality $\operatorname{rank} \beta$. Choose an element $y_{0}$ of $\langle Y\rangle$. Since $A$ is a basis algebra, there are idempotent endomorphisms $\varepsilon, \eta$ such that, for $z \in Z$,

$$
z \varepsilon=\left\{\begin{array}{ll}
z & \text { if } z \in X \\
y_{0} & \text { otherwise }
\end{array} \quad \text { and } \quad z \eta= \begin{cases}z & \text { if } z \in Y \\
y_{0} & \text { otherwise }\end{cases}\right.
$$

It is clear that $\operatorname{rank} \varepsilon=|X| \operatorname{rank} \eta=|Y|$ and $\eta \varepsilon=\eta$. Since $\operatorname{rank} \varepsilon=\operatorname{rank} \alpha$, we have $\varepsilon \in I$, and so, since $I$ is an ideal, $\eta \in I$. Then $\beta \in I$ because $\operatorname{rank} \beta=\operatorname{rank} \eta$.

It follows that if $I \neq$ End $A$ and $\mu$ is the least cardinal such that there is an endomorphism of rank $\mu$ which is not in $I$, then $I=T_{\mu}$.

For aesthetic reasons, we denote the $*$-ideal $T_{n+1}$ by $S_{n}$ for all $n \in \mathbb{N} \cup\{0\}$.

We briefly consider the set $S_{0}$ of endomorphisms of rank 0 , that is, those with image $\langle\emptyset\rangle$. Of course, if $\langle\emptyset\rangle=\emptyset$, then $S_{0}=\emptyset$. On the other hand, if $c \in\langle\emptyset\rangle$ and $X$ is a basis for $A$, then there is an endomorphism $\alpha$ with $X \alpha=c$ so that $\operatorname{Im} \alpha=\langle\emptyset\rangle$ and $\alpha \in S_{0}$. Thus we have the first part of the following lemma.

Lemma 7.2. Let $A$ be a basis algebra. Then $S_{0} \neq \emptyset$ if and only if $\langle\emptyset\rangle \neq \emptyset$. When $S_{0} \neq \emptyset$, it is a left zero semigroup.

Proof. Suppose that $\langle\emptyset\rangle \neq \emptyset$. Let $B$ be any subalgebra with rank 0 . Then $\mathrm{PC}(B)=\mathrm{PC}(\emptyset)$ so that for any $b \in B, x \prec \emptyset$. From the comments following the definition of the relation $\prec$, we have $x \in\langle\emptyset\rangle$. Thus $B=\langle\emptyset\rangle$. Now for any $\alpha, \beta \in S_{0}, \operatorname{Im} \alpha=\langle\emptyset\rangle$ and $c \beta=c$ for all $c \in\langle\emptyset\rangle$, so $\alpha \beta=\alpha$ and $S_{0}$ is a left zero semigroup. 
We now examine more closely the Rees quotients $S_{n} / S_{n-1}$ for $n \in \mathbb{N}$.

Proposition 7.3. Let $n \in \mathbb{N}$ and let $A$ be a stable basis algebra of rank at least $n$. Then $S_{n} / S_{n-1}$ is a primitive abundant semigroup in which all the non-zero idempotents are $\mathscr{D}$ related.

Proof. We already know that $S_{n} / S_{n-1}$ is abundant, has only one non-zero $\mathscr{D}^{*}$-class and that every $\mathscr{H}^{*}$-class contains a regular element. Let $\varepsilon, \eta$ be non-zero idempotents. Since they are $\mathscr{D}^{*}$-related and $\mathscr{D}^{*}=\mathscr{R}^{*} \circ \mathscr{L}^{*}$, there is an endomorphism $\alpha$ such that $\varepsilon \mathscr{R}^{*} \alpha \mathscr{L}^{*} \eta$. Now every $\mathscr{H}^{*}$-class contains a regular element, and so we may assume that $\alpha$ is regular. Then we have $\varepsilon \mathscr{R} \alpha \mathscr{L} \eta$, that is, $\varepsilon \mathscr{D} \eta$.

Now let $\epsilon, \eta$ be non-zero idempotents of $S_{n} / S_{n-1}$ with $\epsilon \eta=\eta=\eta \epsilon$. Then $\operatorname{Im} \eta=\operatorname{Im} \eta \epsilon \subseteq$ $\operatorname{Im} \epsilon$ and by Corollary 4.4, $\operatorname{Im} \eta$ and $\operatorname{Im} \epsilon$ are pure, so that $\operatorname{Im} \eta$ has a basis $X$ with can be extended to a basis $Y$ of $\operatorname{Im} \epsilon$. But $|X|=|Y|$ so that $X=Y$ and $\operatorname{Im} \epsilon=\operatorname{Im} \eta$. Thus by Corollary 4.6, $\epsilon \mathscr{L}^{*} \eta$ so that $\eta=\epsilon \eta=\epsilon$. Thus the non-zero idempotents are primitive.

It follows from Proposition 7.3 together with Proposition 4.8 and Theorem 4.9 of [6] that $S_{n} / S_{n-1}$ is isomorphic to a Rees matrix semigroup $\mathscr{M}^{0}(S ; I, \Lambda ; P)$ where $S$ is a cancellative monoid, and each row and each column of the sandwich matrix $P$ contains a unit of $S$. Our next goal is to find appropriate $S, I, \Lambda$ and $P$, and to give an explicit isomorphism.

Let $n \in \mathbb{N}$ and $A$ be a stable basis algebra of rank at least $n$. We index the set of kernels of endomorphisms of $A$ of rank $n$ by $I$, and the set of pure subalgebras of $A$ of rank $n$ by $\Lambda$, so that we can write the kernels as $\rho_{i}$ for $i \in I$ and the pure subalgebras as $B_{\lambda}$ for $\lambda \in \Lambda$. In view of Lemmas 4.2 and 4.6, we have, in effect, indexed the $\mathscr{R}^{*}$-classes of $S_{n} / S_{n-1}$ by $I$ and the $\mathscr{L}^{*}$-classes of $S_{n} / S_{n-1}$ by $\Lambda$. We write $H_{i \lambda}^{*}$ for the $\mathscr{H}^{*}$-class $R_{i}^{*} \cap L_{\lambda}^{*}$.

Choose a particular element $\lambda_{0}$ of $\Lambda$ and put $B=B_{\lambda_{0}}$. Then $B$ is a stable basis algebra of rank $n$. Let $S$ be the $\mathscr{H}^{*}$-class of the identity of End $B$, so that $S$ consists of the one-one endomorphisms of $B$ of rank $n$. We know by Lemma 1.12 of [6] that $S$ is a cancellative submonoid of End $B$.

Let $\varepsilon$ be an idempotent of End $A$ with $\operatorname{Im} \varepsilon=B$; then $H_{\varepsilon}^{*}=H_{i_{0} \lambda_{0}}^{*}$ for some element $i_{0}$ of I. Also, $H_{\varepsilon}^{*}$ consists of those endomorphisms $\alpha$ of $A$ with $\mathrm{PC}(\operatorname{Im} \alpha)=B$ and $\operatorname{Ker} \alpha=\operatorname{Ker} \varepsilon$. Hence if $\alpha \in H_{\varepsilon}^{*}$, then $\alpha$ is one-one on $B$. Also, since $\varepsilon \alpha=\alpha$, we have

$$
\operatorname{rank}\left(\left.\alpha\right|_{B}\right)=\operatorname{rank}((\operatorname{Im} \varepsilon) \alpha)=\operatorname{rank}(\operatorname{Im} \varepsilon \alpha)=\operatorname{rank} \alpha=n .
$$

Thus $\left.\alpha\right|_{B} \in S$, and so we can define $\theta: H_{\varepsilon}^{*} \rightarrow S$ by $\alpha \theta=\left.\alpha\right|_{B}$. Clearly, $\theta$ is a morphism, and if $\alpha \theta=\beta \theta$, then $\alpha$ and $\beta$ agree on $\operatorname{Im} \varepsilon$, so that $\alpha=\varepsilon \alpha=\varepsilon \beta=\beta$, and $\theta$ is one-one. If $\delta \in S$, then $\varepsilon \delta \in$ End $A$ and $(\varepsilon \delta) \theta=\delta$ so that $\theta$ is an isomorphism.

We now turn our attention to the sandwich matrix. Put $H^{*}=H_{\varepsilon}^{*}=H_{i_{0} \lambda_{0}}^{*}$. For each $i \in I$, we choose a regular element $r_{i}$ in $H_{i \lambda_{0}}^{*}$, and, for each $\lambda \in \Lambda$, we choose a regular element $q_{\lambda}$ in $H_{i_{0} \lambda}^{*}$. Such choices are possible, in view of Theorem 4.9. Moreover, by Proposition 1.13 of [6], the mapping $x \mapsto r_{i} x$ is a bijection from $H^{*}$ onto $H_{i \lambda_{0}}^{*}$, and $y \mapsto y q_{\lambda}$ is a bijection from $H_{i \lambda_{0}}^{*}$ onto $H_{i \lambda}^{*}$. Thus, once we have chosen the $r_{i}$ and the $q_{\lambda}$, we have a unique expression $r_{i} a q_{\lambda}$ for each element of $H_{i \lambda}^{*}$ where $a \in H^{*}, i \in I$ and $\lambda \in \Lambda$. 
We now define $P$ to be the $\Lambda \times I$ matrix $\left(p_{\lambda i}\right)$ where $p_{\lambda i}=q_{\lambda} r_{i}$ for $(i, \lambda) \in I \times \Lambda$. Then either $p_{\lambda i}=0$ or, by [6, Lemma 3.3], $p_{\lambda i} \in H^{*}$. Furthermore, as in the proof of Theorem 3.8 of [6], we find that, for our case, each row and column contains a unit of $H^{*}$.

We can now construct a Rees matrix semigroup $\mathscr{M}^{0}=\mathscr{M}^{0}\left(H^{*} ; I, \Lambda ; P\right)$, and, from the proof of [6, Theorem 3.8], we have that the map $\varphi: \mathscr{M}^{0} \rightarrow$ End $A$, defined by $0 \varphi=0$ and $(i, a, \lambda) \varphi=r_{i} a q_{\lambda}$, is an isomorphism.

Now, for each $(i, \lambda) \in I \times \Lambda$, where $p_{\lambda i} \in H^{*}$, let $q_{\lambda i}=p_{\lambda i} \theta$, that is, $q_{\lambda i}$ is the restriction of $p_{\lambda i}$ to $B$, and $q_{\lambda i} \in S$, and put $q_{\lambda i}=0$ if $p_{\lambda i}=0$. Hence we can form the Rees matrix semigroup $\mathscr{M}_{1}^{0}=\mathscr{M}^{0}(S ; I, \Lambda ; Q)$ where $Q$ is the $\Lambda \times I$ matrix $\left(q_{\lambda i}\right)$. It is straightforward to verify that the mapping $\psi: \mathscr{M}^{0} \rightarrow \mathscr{M}_{1}^{0}$ given by $0 \psi=0$ and $(i, a, \lambda) \psi=(i, a \theta, \lambda)$, is an isomorphism. Thus we have now proved the following result.

Theorem 7.4. Let $n \in \mathbb{N}$ and let $A$ be a stable basis algebra of rank at least $n$. Let $B$ be a pure subalgebra of $A$ with $\operatorname{rank} B=n$, and let $S$ be the $\mathscr{H}^{*}$-class of the identity of End $B$. Then there are sets $I, \Lambda$ and a $\Lambda \times I$ matrix $Q$ over $S \cup\{0\}$ such that $S_{n} / S_{n-1}$ is isomorphic to the Rees matrix semigroup $\mathscr{M}^{0}(S ; I, \Lambda ; Q)$.

We remark that it follows from Theorem 6.12 that each $S_{n} / S_{n-1}$ is a left order in a completely 0-simple semigroup. Since $S_{n} / S_{n-1}$ is abundant, this completely 0-simple semigroup of left quotients is unique, and it follows from Theorem 7.4 that it is isomorphic to $\mathscr{M}^{0}(G ; I, \Lambda ; Q)$ where $G$ is the group of left quotients of $S$.

\section{REFERENCES}

[1] S. Armstrong, Structure of concordant semigroups, J. Algebra 118 (1988), 205-260.

[2] J.A. Beachy, Introductory Lectures on Rings and Modules, Cambridge University Press, Cambridge, 1999.

[3] P.J. Cameron and C. Szabó, Independence algebras, J. London Math. Soc., 61 (2000), 321-334.

[4] P. M. Cohn, Universal Algebra, Harper \& Row, New York, 1965.

[5] A. El Qallali and J. Fountain, Idempotent-connected abundant semigroups, Proc. Roy. Soc. Edinburgh 91A (1981), 79-90.

[6] J. Fountain, Abundant semigroups, Proc. London Math. Soc. 44 (1982), 103-129.

[7] J. Fountain, Products of idempotent integer matrices, Math. Proc. Camb. Phil. Soc. 110 (1991), 431-441.

[8] J. Fountain and V. Gould, Orders in rings without identity, Commun. Algebra 18 (1990), 3085-3110.

[9] J. Fountain and V. Gould, Straight left orders in rings, Quart. J. Math. Oxford 43 (1992), 303-311.

[10] J. Fountain and V. Gould, Relatively free algebras with weak exchange properties, http://wwwusers.york. ac.uk/ varg1.

[11] J. Fountain and V. Gould, Relatively free algebras with weak exchange properties, J. Austral. Math. Soc. 75 (2003), 355-384.

[12] J. Fountain and V. Gould, Products of idempotent endomorphisms of relatively free algebras with weak exchange properties, preprint 2003.

[13] V. Gould, Semigroups of left quotients - the uniqueness problem, Proc. Edinburgh Math. Soc. 35 (1992), 213-226.

[14] V. Gould, Independence algebras, Algebra Universalis 33 (1995), 294-318.

[15] V. Gould, Semigroups of left quotients; existence, straightness and locality, J. Algebra 267 (2003), $514-541$.

[16] V. Gould, Abundant and ample straight left orders, Period. Math. Hungar. 46 (2003), 171-179. 
[17] G. Grätzer, Universal Algebra, Van Nostrand, Princeton, N.J., 1968.

[18] J. M. Howie, Fundamentals of Semigroup Theory, Oxford University Press, Oxford, 1995.

[19] T. J. Laffey, Products of idempotent matrices, Linear and Multilinear Algebra, 14 (1983), 309-314.

[20] E.S. Lyapin, Semigroups, Amer. Math. Soc., Providence R.I. 1963.

[21] M. Kilp, U. Knauer and A.V. Mikhalev, Monoids, Acts and Categories, Walter de Gruyter, Berlin, 2000.

[22] M.V. Lawson, The Structure Theory of Abundant Semigroups, D.Phil. thesis, York, 1985.

[23] R. N. McKenzie, G. F. McNulty, and W. T. Taylor, Algebra, Lattices, Varieties, Wadsworth, 1983.

[24] W. Narkiewicz, Independence in a certain class of abstract algebras, Fund. Math. 50 (1961/62), 333-340.

[25] W. Ruitenburg, Products of idempotent matrices over Hermite domains, Semigroup Forum, 46 (1993), $371-378$.

Department of Mathematics, University of York, Heslington, York YO10 5DD, UK E-mail address: jbf1@york.ac.uk

Department of Mathematics, University of York, Heslington, York YO10 5DD, UK

E-mail address: varg1@york.ac.uk 